University of Warwick institutional repository: http://go.warwick.ac.uk/wrap

This paper is made available online in accordance with publisher policies. Please scroll down to view the document itself. Please refer to the repository record for this item and our policy information available from the repository home page for further information.

To see the final version of this paper please visit the publisher's website. Access to the published version may require a subscription.

Author(s): Kowalczyk, R. M., Kemp, T. F., Walker, D., et al.

Article Title: A variable temperature solid-state nuclear magnetic resonance, electron paramagnetic resonance and Raman scattering study of molecular dynamics in ferroelectric fluorides

Year of publication: 2011

Link to published article : http://dx.doi.org/10.1088/09538984/23/31/315402

Publisher statement: (c IOP. Kowalczyk, R. M., Kemp, T. F., Walker, D., et al. (2011). A variable temperature solid-state nuclear magnetic resonance, electron paramagnetic resonance and Raman scattering study of molecular dynamics in ferroelectric fluorides. Journal of Physics: Condensed Matter, 23(31), article 315402. This is an authorcreated, un-copyedited version of an article accepted for publication in Journal of Physics: Condensed Matter. IOP Publishing Ltd is not responsible for any errors or omissions in this version of the manuscript or any version derived from it. The definitive publisher-authenticated version is available online at http://dx.doi.org/10.1088/09538984/23/31/315402 


\title{
A variable temperature solid-state Nuclear Magnetic Resonance, Electron Paramagnetic Resonance and Raman scattering study of molecular dynamics in ferroelectric \\ fluorides
}

\author{
Radoslaw M Kowalczyk ${ }^{1,2 \# *}$, Thomas F Kemp ${ }^{1}$, David Walker ${ }^{1}$, Kevin J Pike ${ }^{1}$, \\ Pamela A Thomas $^{1}$, Jens Kreisel ${ }^{2}$, John V Hanna ${ }^{1}$, Ray Dupree ${ }^{1}$, Mark E \\ Newton $^{1}$, Mark E Smith ${ }^{1}$ \\ ${ }^{1}$ Department of Physics, University of Warwick, Gibbet Hill Road, Coventry CV4 \\ 7AL, UK \\ ${ }^{2}$ Laboratoire des Matériaux et du Génie Physique, Miniatec, CNRS, Grenoble \\ Institute of Technology, 3 parvis Louis Néel, 38016 Grenoble, France \\ E-mail: r.kowalczyk@ surrey.ac.uk
}

\begin{abstract}
The local nuclear and electronic structures and molecular dynamics of the ferroelectric lattice in selected geometric fluorides $\left(\mathrm{BaMgF}_{4}, \mathrm{BaZnF}_{4}, \mathrm{BaMg}_{1-\mathrm{x}} \mathrm{Mn}_{\mathrm{x}} \mathrm{F}_{4}\right.$ and $\mathrm{BaMg}_{1-\mathrm{x}} \mathrm{Ni}_{\mathrm{x}} \mathrm{F}_{4} ; \mathrm{x}=0.001$ and 0.005 ) has been investigated. The ${ }^{19} \mathrm{~F}$ and ${ }^{25} \mathrm{Mg}$ isotropic chemical shift $\delta_{\text {iso, }}{ }^{25} \mathrm{Mg}$ quadrupolar coupling constants $\left(\mathrm{C}_{\mathrm{q}}\right)$ and asymmetry parameters $(\eta)$ reflect the geometry of the coordination spheres. Zero Field Splitting parameters $|D|$ and $|E|$ are consistent with distorted axial symmetry (low temperatures) and nearly rhombic (high temperatures) of octahedral $\mathrm{Mn}^{2+}$ coordination. High resolution of NMR, EPR and phonon spectra are consistent with the highly ordered crystallographic structure. Combined multitechniques evidence of the subtle discontinuous changes in temperature dependencies of $|D|$ and $|E|$, isotropic chemical shifts $\delta_{\text {iso }}$ and signature parameters of Raman bands and suggest a discontinuous structural distortion of the fluoride octahedra. The temperature at which this change occurs depends on the ionic radius of the central ion of the octahedral site and it is estimated at $\sim 300 \mathrm{~K}$ for $\mathrm{Zn}^{2+}$ fluorides and to $\sim 240 \mathrm{~K}$ for $\mathrm{Mg}^{2+}$ fluorides. This geometrical distortion modifies lattice dynamics and originates in rotation of the fluoride octahedra around a new direction approximately perpendicular to that related to the paraelectric - ferroelectric phase transition.
\end{abstract}

\footnotetext{
\# Present address: Department of Physics, University of Surrey, Guildford GU2 7XH, UK

* To whom correspondence should be sent
} 
Key words: geometric ferroelecrics, multiferroics, solid-state MAS NMR, magnesium NMR, EPR, Raman scattering

\section{PACS code: Spectroscopy of solid state dynamics 78.47-p}

Submitted to: Journal of Physics: Condensed Matter

\section{Introduction}

Ferroelectrics and ferromagnets are of great technological importance and their properties are intimately determined by their structure and the dynamics at the atomic and molecular levels [1-3]. In multiferroic materials both electric and magnetic orders coexist and can interact [4], despite the fact that the well accepted mechanisms of electric and magnetic orderings have been regarded in the past as mutually exclusive [5]. Some multiferroics combine new types of ferroelectricity, as for instance in geometrical ferroelectrics (where electric polarisation arises as a consequence of atom shifts and structural distortions) together with magnetism $[6,7]$.

Despite a recent resurgence of interest in geometric ferroelectrics, our understanding of the molecular dynamics in such ferroelectrics remains limited [8]. In particular, the influence of geometric distortions on the lattice dynamics deserve further experimental attention as these are often related to the cation displacement responsible for electric polarisation [9]. One of the most intensely discussed problems is coupling between magnetism and lattice dynamics [10]; in this investigation magnetic ions are present as both diluted dopants and as ions responsible for magnetic ordering in the sample. An improved understanding of molecular dynamics is also crucial in order to understand the stability of the polarisation and magnetisation in multiferroics with geometric feroelectricity, which potentially could result in greater macroscopic control of both magnetic and electric orders being achieved in these systems [11].

Ferroelectric, optical and multiferroic properties of geometric fluorides have been studied and characterized in detail previously [12-17]. This study focuses on the diamagnetic members of the BaMF 4 family $\left(\mathrm{M}^{2+}=\mathrm{Mn}^{2+}, \mathrm{Fe}^{2+}, \mathrm{Co}^{2+}, \mathrm{Ni}^{2+}, \mathrm{Cr}^{2+}, \mathrm{Zn}^{2+}, \mathrm{Mg}^{2+}\right)$, namely $\mathrm{BaMgF}_{4}$ and $\mathrm{BaZnF}_{4}$. They were subsequently doped with $\mathrm{Mn}^{2+}$ and $\mathrm{Ni}^{2+}$ ions to introduce paramagnetic probes, identical to that responsible for magnetism in $\mathrm{BaMnF}_{4}$ and $\mathrm{BaNiF}_{4}$, and to allowed other studies to be facilitated [18, 19]. For three members of the $\mathrm{BaMF}_{4}$ family, namely $\mathrm{BaMnF}_{4} \mathrm{BaCoF}_{4}$ and $\mathrm{BaNiF}_{4}$, multiferroic properties were reported and are now widely accepted [13-15]. The molecular dynamics of geometric fluorites have also attracted attention, but most studies are limited to understanding the origin of spontaneous polarisation and the paraelectric - ferroelectric phase transition, which occurs around 100 $\mathrm{K}$ above the melting point (in the range of 990 to $1240 \mathrm{~K}$, respectively for $\mathrm{BaFeF}_{4}$ to $\mathrm{BaNiF}_{4}$ ) [20, 21]. The low temperature studies have been predominantly focused on magnetic properties of $\mathrm{BaMnF}_{4}$ and $\mathrm{BaNiF}_{4}[12,15,22,23]$. The incommensurate - commensurate phase transition, which 
occurs at $250 \mathrm{~K}$ has been observed and accepted only in $\mathrm{BaMnF}_{4}[12,24]$. Only recently, low temperature dynamics in $\mathrm{BaZnF}_{4}$ and $\mathrm{BaMn}_{1-\mathrm{x}} \mathrm{Zn}_{\mathrm{x}} \mathrm{F}_{4}$ have been studied and analysed in more detail [25-28].

Magnetic resonance techniques have been quite rarely used to study these fluorides despite the possibility of providing information on the temperature changes in the crystal field induced by the lattice dynamics $[12,29]$. Solid-state ${ }^{19} \mathrm{~F}$ NMR was used to provide a reference for quantum mechanical calculation of the chemical shifts in fluorides [30], whereas EPR studies have been focused on characterisation of properties and the coordination sphere of paramagnetic ions in the lattice [31, 32]. Only one study uses paramagnetic $\mathrm{Mn}^{2+}$ as a probe doped in a $\mathrm{BaMgF}_{4}$ matrix [33]. The directions of the Zero Field Splitting (ZFS) tensor, evaluated at a few temperatures, were used to define and to monitor an order parameter for the paraelectric - ferroelectric phase transition and to estimate the Curie temperature [33], however low temperature molecular dynamics and the direct dependence of ZFS on temperature were not explored [33].

Raman experiments were also mostly focused on the ferroelectric - paraelectric phase transition [12, 34] and the incommensurate - commensurate phase transition in $\mathrm{BaMnF}_{4}$ [24]. Detailed data over a wide range of temperatures have been published only for $\mathrm{BaZnF}_{4}$ and there are reports of anomalous behaviour for some particular modes at $300 \mathrm{~K}$ [25]. Such behaviour was interpreted as dynamic order - disorder changes, while a phase transition does not occur [25-27]. This hypothesis has attracted some attention and studies of $\mathrm{BaMn}_{1-\mathrm{x}} \mathrm{Zn}_{\mathrm{x}} \mathrm{F}_{4}(0<\mathrm{x}<0.25)$ soon followed, but without providing a definitive model [28].

The motivation of this work is to extend our knowledge and understanding of the lattice dynamics in geometric ferroelectrics using a variable temperature approach for a suite of different experimental methods. Solid-state Nuclear Magnetic Resonance (solid-state NMR) and Electron Paramagnetic Resonance (EPR) provide local nuclear and electronic probes of the lattice in different microenvironments of each system, while Raman scattering captures the characteristic of local correlations of phonons. The choice of these techniques is justified by their past successful application for studying molecular dynamics and phase transitions in a variety of inorganic materials [35-37]. They also provide the possibility to study a comprehensive set of materials including diamagnetic (NMR), magnetically diluted (EPR) and magnetic samples (Raman) [38-40]. This study re-examines data from $\mathrm{BaZnF}_{4}\left(\mathrm{NMR}\right.$ and Raman) and extends the study to $\mathrm{BaZn}_{0.999} \mathrm{Mn}_{0.001} \mathrm{~F}_{4}$ (EPR) to obtain a picture of molecular dynamic changes over a wide range of temperatures. To explore further low temperature molecular dynamics, the main studies of this work have been focused on $\mathrm{BaMgF}_{4}$, $\mathrm{BaMg}_{0.999} \mathrm{Mn}_{0.001} \mathrm{~F}_{4}, \mathrm{BaMg}_{0.995} \mathrm{Mn}_{0.005} \mathrm{~F}_{4}, \mathrm{BaMg}_{0.995} \mathrm{Ni}_{0.005} \mathrm{~F}_{4}$ (NMR, EPR, Raman extended to energies above $200 \mathrm{~cm}^{-1}$ ). Indeed, if the picture in fluorites is universal as suggested in [25], dynamic order 


\section{Molecular dynamics in geometric ferroelectric fluorides}

should also be even more apparent in $\mathrm{BaMgF}_{4}$ because of a lower level of structural distortion due to the smaller radius of $\mathrm{Mg}^{2+}$ compared to $\mathrm{Zn}^{2+}$.

This paper reports experimental data that favours a discontinuous change in structural distortion of fluoride octahedra based on observed temperature changes in the Zero Field Splitting parameters $|D|$ and $|E|$ extracted from EPR spectra. These changes are supported by discontinuities in the temperature dependence of the isotropic chemical shift $\delta_{\text {iso }}$ in variable temperature solid-state magic angle spinning (MAS) NMR spectra and by observed changes in frequencies and linewidths of some high frequency bands in the Raman spectra. This geometrical change is expected to play an important role in modifying lattice dynamics as an initial step in the creation of dynamic order in the structure.

\section{Experimental procedures}

\subsection{Synthesis and structure}

$\mathrm{BaMgF}_{4}$ and $\mathrm{BaZnF}_{4}$ were synthesized by reacting mixtures of fine powders of binary compounds $\left(\mathrm{BaF}_{2}\right.$ and $\mathrm{MgF}_{2}$ or $\left.\mathrm{ZnF}_{2}\right)$ in graphite crucibles under an $\mathrm{Ar}$ atmosphere. The synthesis temperature was ca. $50-100 \mathrm{~K}$ above the melting point of $\mathrm{BaMgF}_{4}$ or $\mathrm{BaZnF}_{4}$ but below the melting point of the reactants. The best samples were obtained by allowing 48 hours for the reaction followed by a slow cooling at a rate of $2 \mathrm{~K} /$ hour. The reaction time and cooling rate were carefully controlled to avoid creation of different temperature-related phases because there was a narrow temperature window for synthesis. These synthesis conditions led to a shell containing small single crystals. The outer layer of the shell contained a small amount of oxide impurities as a result of the reaction of water trapped in $\mathrm{MgF}_{2}$ or especially in highly hygroscopic $\mathrm{ZnF}_{2}$. Only single crystals from the inside shell were selected as samples of appropriate purity.

The synthesized prototype compounds $\mathrm{BaMgF}_{4}$ and $\mathrm{BaZnF}_{4}$ were subsequently doped with a low level $(0.1 \%$ and $0.5 \%)$ of paramagnetic $\mathrm{Mn}^{2+}$ and $\mathrm{Ni}^{2+}$ ions, which substitute for the $\mathrm{Mg}^{2+}$ and $\mathrm{Zn}^{2+}$ ions in the structure [31]. The synthesis temperature of the doped compounds was adjusted to reflect the lower or higher melting temperature of $\mathrm{MnF}_{2}$ and $\mathrm{NiF}_{2}$ but the general procedure was identical to that used for $\mathrm{BaMgF}_{4}$ or $\mathrm{BaZnF}_{4}$.

The crystallographic structures of synthesized materials were found to be orthorhombic with space group Cmc2 ${ }_{1}$ (by both single crystal and powder X-Ray Diffraction - XRD) in agreement with previously reported data $[18,41]$.

\subsection{Solid-state static and Magic Angle Spinning (MAS) Nuclear Magnetic Resonance (NMR)}


Room temperature solid-state MAS NMR measurements were performed on a Bruker Avance II 500 MHz (11.7 T) spectrometer. A high-speed $2.5 \mathrm{~mm}$ Bruker MAS fluorine background-free probe, tuned to $470.39 \mathrm{MHz}$ was used in association with this spectrometer. All spectra were referenced to an external standard polytetrafluoroethylene (PTFE) at $-123.2 \mathrm{ppm}$. A single $90^{\circ}$ pulse of $4.5 \mu$ s was used. A typical signal consists of 64 accumulated acquisitions. The delay time between acquisitions is typically $5 \mathrm{~s}$. The spinning rates varied between 8 and $30 \mathrm{kHz}$. Variable temperature solid-state MAS NMR measurements were carried out on Varian $600 \mathrm{MHz}(14.1 \mathrm{~T})$ spectrometer. A specially made low-temperature Doty Scientific MAS NMR probe, tuned to $564.29 \mathrm{MHz}$ was used. A single $90^{\circ}$ pulse of $6.0 \mu \mathrm{s}$ was used. A typical signal consists of 16 added acquisitions. Spectra were recorded in the temperature range 120 to $310 \mathrm{~K}$. The delay time between acquisitions is typically 10 to $30 \mathrm{~s}$ depending on temperature. The spinning rates varied between 8 and $16 \mathrm{kHz}$, at low and room temperature respectively.

Static broadline ${ }^{25} \mathrm{Mg}$ NMR data were acquired at ambient temperature $(292 \mathrm{~K})$ and at $150 \mathrm{~K}$ using a Varian $600 \mathrm{MHz}(14.1 \mathrm{~T})$ spectrometer operating at a ${ }^{25} \mathrm{Mg}$ Larmor frequency of $36.72 \mathrm{MHz}$. A Bruker static-NMR probe with $10 \mathrm{~mm}$ diameter coil was used with a flow of nitrogen gas to cool the sample. Spectra were acquired with a pulse sequence $\theta_{\pi / 2}-\tau-\theta_{\pi}-\tau_{1}$ - (FID acqusition) [42, 43]. A non-selective $\pi / 2$ pulse widths of $15 \mu \mathrm{s}\left(\gamma_{\mathrm{Mg}} B_{1} / 2 \pi=16.7 \mathrm{kHz}\right)$ was calibrated using solid $\mathrm{MgO}$ and used to calculate selective 'solid' pulses, with $\theta_{\pi / 2}=4 \mu$ s and $\theta_{\pi}=8 \mu$ s used for experiments at both temperatures. The $\tau$ echo delay was $50 \mu \mathrm{s}$. $8000 \mathrm{FID}$ transients were averaged at $292 \mathrm{~K}$ with $20 \mathrm{~s}$ relaxation delays and 80 were averaged at $150 \mathrm{~K}$ with $120 \mathrm{~s}$ delays. The spectra were referenced using the ${ }^{25} \mathrm{Mg}$ resonance at $26 \mathrm{ppm}$ for soild $\mathrm{MgO}$ at $292 \mathrm{~K}$.

All solid-state MAS NMR spectra were simulated using the free-distribution simulation program SIMPSON [44]. Quantum mechanical calculations of chemical shifts and chemical shift anisotropies have been carried out using plane-wave basis sets and periodic boundary conditions based on experimental XRD data (CASTEP, [45]). The calculated values of chemical shielding were referenced (uniformly shifted), to fit the best experimental results.

\subsection{Electron Paramagnetic Resonance (EPR)}

The variable temperature continuous wave Electron Paramagnetic Resonance (EPR) experiments were performed using a Bruker EMX spectrometer consisting of an electromagnet and X-band (9.450 $\mathrm{GHz}$ ) microwave bridge ER 041XG with Bruker ER4105D double rectangular resonator. A heliumgas flow cryostat was used and the temperature was varied in the range 5 to $300 \mathrm{~K}( \pm 1 \mathrm{~K})$ by an Oxford Instruments auto-tuning temperature controller ITC503. Control experiments were carried out with a Bruker ER 4102ST rectangular resonator with a nitrogen-gas flow cryostat in the temperature range 120 to $360 \mathrm{~K}$ monitored by an Oxford Instruments auto-tuning temperature controller. High 


\section{Molecular dynamics in geometric ferroelectric fluorides}

temperature experiments in the range 300 to $670 \mathrm{~K}$ were performed using a modified Bruker hightemperature cavity and nitrogen-gas flow system. The Q-band (32.28 GHz) EPR spectra were recorded using a Bruker ELEXYS spectrometer and SuperQ-FT microwave bridge in conjunction with a Bruker Q-band EN517D2 resonator. All spectra were recorded with $100 \mathrm{kHz}$ modulation frequency and modulation amplitudes in the range 0.1 to $0.3 \mathrm{mT}$, with the exception of hightemperature measurements where the maximum available modulation amplitude of $0.02 \mathrm{mT}$ was applied. A typical microwave power of $1.00 \mathrm{~mW}$ was used at which no saturation effects were observed. All EPR spectra were simulated using the EASYSPIN simulation package [46].

\subsection{Raman spectroscopy}

Raman spectra were recorded in a back-scattering geometry using a LabRam Jobin - Yvon spectrometer with spectral resolution of about $0.2 \mathrm{~cm}^{-1}$ and a Rayleigh scattering cut-off at $80 \mathrm{~cm}^{-1}$ with Notch filter. The laser beam of an $\mathrm{Ar}^{+}$ion laser of $488 \mathrm{~nm}$ was focused to a $1 \mu \mathrm{m}^{2}$ spot through a times 50 long focal objective. All experiments were performed with a typical incident power of 4 to 8 $\mathrm{mW}$, after verification that this power level does not incur sample damage. Temperature-dependent measurements were carried out on selected good-quality fluoride crystals by using a commercial Linkam cooling/heating stage and were conducted upon heating with a typically slow rate of $10 \mathrm{~K} /$ min. The deconvolution of the Raman spectra was performed using the LabSpec software.

\section{Results and discussion}

The microstructure of $\mathrm{BaMF}_{4}\left(\mathrm{M}^{2+}=\mathrm{Mn}^{2+}, \mathrm{Fe}^{2+}, \mathrm{Co}^{2+}, \mathrm{Ni}^{2+}, \mathrm{Cr}^{2+}, \mathrm{Zn}^{2+}, \mathrm{Mg}^{2+}\right)$ consists of a network of octahedra with a central $\mathrm{M}^{2+}$ ion and six nearest-neighbour fluorine ligands ions as shown schematically in figure $1 . \mathrm{Ba}^{2+}$ ions are located in the cavities between octahedra (figure 1). The metal - fluorine distances range from 2.60 to $3.00 \AA$ for $\left(\mathrm{Ba}^{2+}-\mathrm{F}^{-}\right)$and from 1.93 to $2.07 \AA$ for $\left(\mathrm{M}^{2+}-\mathrm{F}^{-}\right)[18$, 19]. 


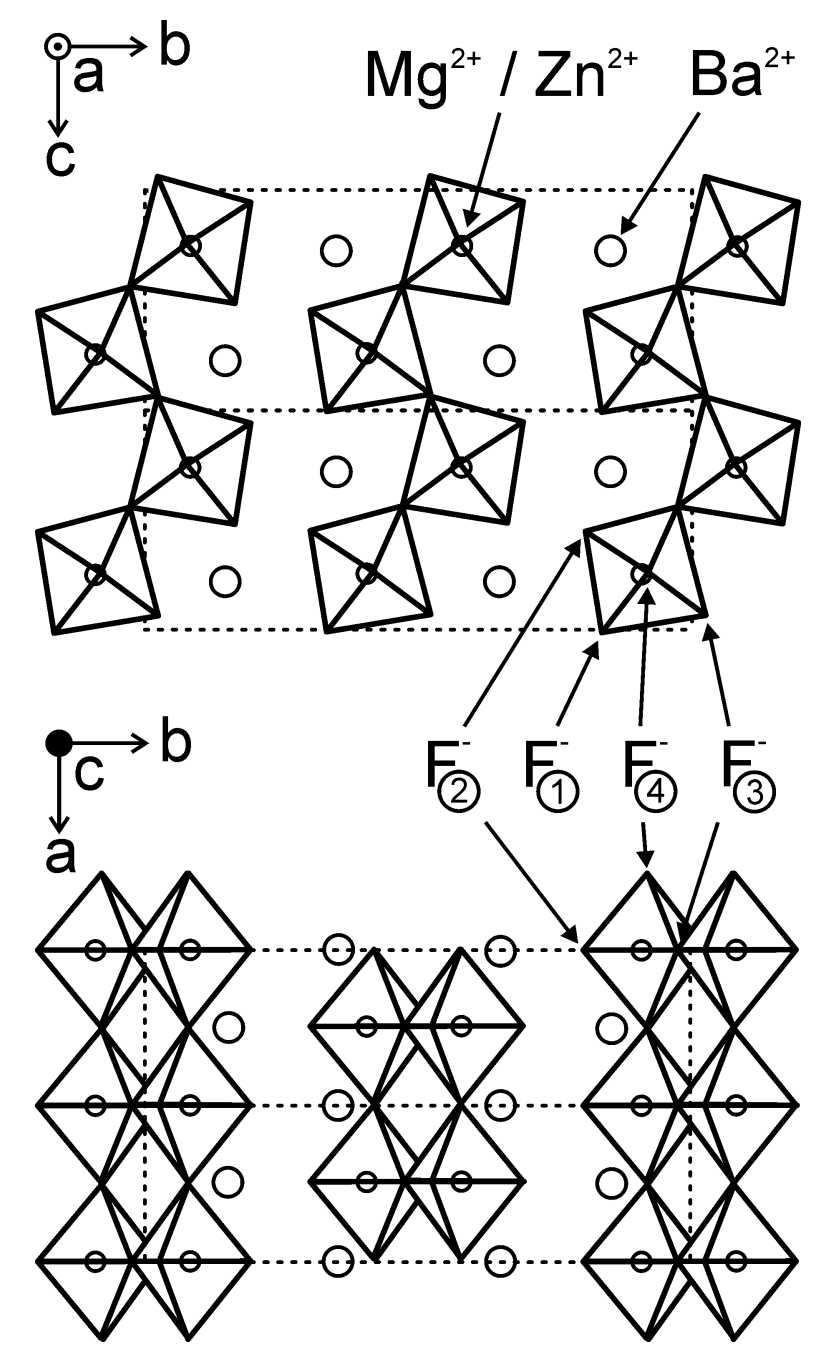

Figure 1. The schematic structure of $\mathrm{BaMgF}_{4}$ and $\mathrm{BaZnF}_{4}$. Fluorine atoms form a distorted octahedron, which surrounds $\mathrm{Mg}^{2+} / \mathrm{Zn}^{2+}$ ions. $\mathrm{Ba}^{2+}$ ions lay between chains of rotated octahedra. The four fluorine nuclei per unit cell are labelled.

\section{$3.1{ }^{19} \mathrm{~F}$ and ${ }^{25} \mathrm{Mg}$ Nuclear Magnetic Resonance (NMR)}

The room temperature ${ }^{19} \mathrm{~F}$ solid-state MAS NMR spectra of diamagnetic $\mathrm{BaZnF}_{4}$ and $\mathrm{BaMgF}_{4}$ acquired at $470.39 \mathrm{MHz}(11.74 \mathrm{~T})$ with a spinning frequency $30 \mathrm{kHz}$ are presented in figure 2(a) and (b) respectively, with the elucidated isotropic chemical shifts $\left(\delta_{\text {iso }}\right)$ collected in table 1 . Each spectrum consists of four peaks which can be attributed to the four inequivalent fluorine nuclei in the unit cell (see figure 1). The upfield ${ }^{19} \mathrm{~F}$ shifts (producing negative $\delta_{\text {iso }}$ values) reflect a significant presence of electron density on each fluorine atom thus corresponding to the highly ionic character of these systems [47].

GIPAW DFT calculations of the ${ }^{19} \mathrm{~F}$ isotropic chemical shifts using the CASTEP code [45] (see Table 1) show that calculated $\delta_{\text {iso }}$ values exhibit general agreement with the experimental measurements. This computational tool also confirmed the assignment of all ${ }^{19} \mathrm{~F} \delta_{\text {iso }}$ to the appropriate fluorine nuclei of the crystallographic structure as presented in figure 1. 


\section{Molecular dynamics in geometric ferroelectric fluorides}

As the main contribution to chemical shift arises from interactions with all the nearest neighbouring atoms, differences in $\delta_{\text {iso }}$ are directly related to distinctive fluorine coordinations between shared (fluorine nuclei $\mathrm{F}(3)$ and $\mathrm{F}(4)$ in figure 1, with two $\mathrm{M}^{2+}$ in the chain) and non-shared (fluorine nuclei $\mathrm{F}(1)$ and $\mathrm{F}(2)$ in figure 1 , with a metal $\mathrm{M}^{2+}$ only on one side) positions in the fluoride octahedral. The difference in $\delta_{\text {iso }}$ between $\mathrm{F}(1)$ and $\mathrm{F}(2)$, as well as $\mathrm{F}(3)$ and $\mathrm{F}(4)$, is more likely due to the differing number of barium atoms in the coordination sphere and as consequence very different local geometry. The shift differences reported between all the sets of sites are in good agreement with previous ${ }^{19} \mathrm{~F}$ measurements [48], with the shifts here being reported with higher precision as a result of the higher field and faster spinning applied.

The relative differences in $\delta_{\text {iso }}$ between $\mathrm{BaZnF}_{4}$ and $\mathrm{BaMgF}_{4}$ are a consequence of the more electronegative character of $\mathrm{Mg}^{2+}$ compared to $\mathrm{Zn}^{2+}$, which then produces the more ionic character of $\mathrm{BaMgF}_{4}$, and the difference in ionic radius between $\mathrm{Mg}^{2+}(\mathrm{r}=0.66 \AA)$ and $\mathrm{Zn}^{2+}(\mathrm{r}=0.74 \AA$ ), which is in turn related to the more distorted octahedra in $\mathrm{BaZnF}_{4}$ at room temperature. 

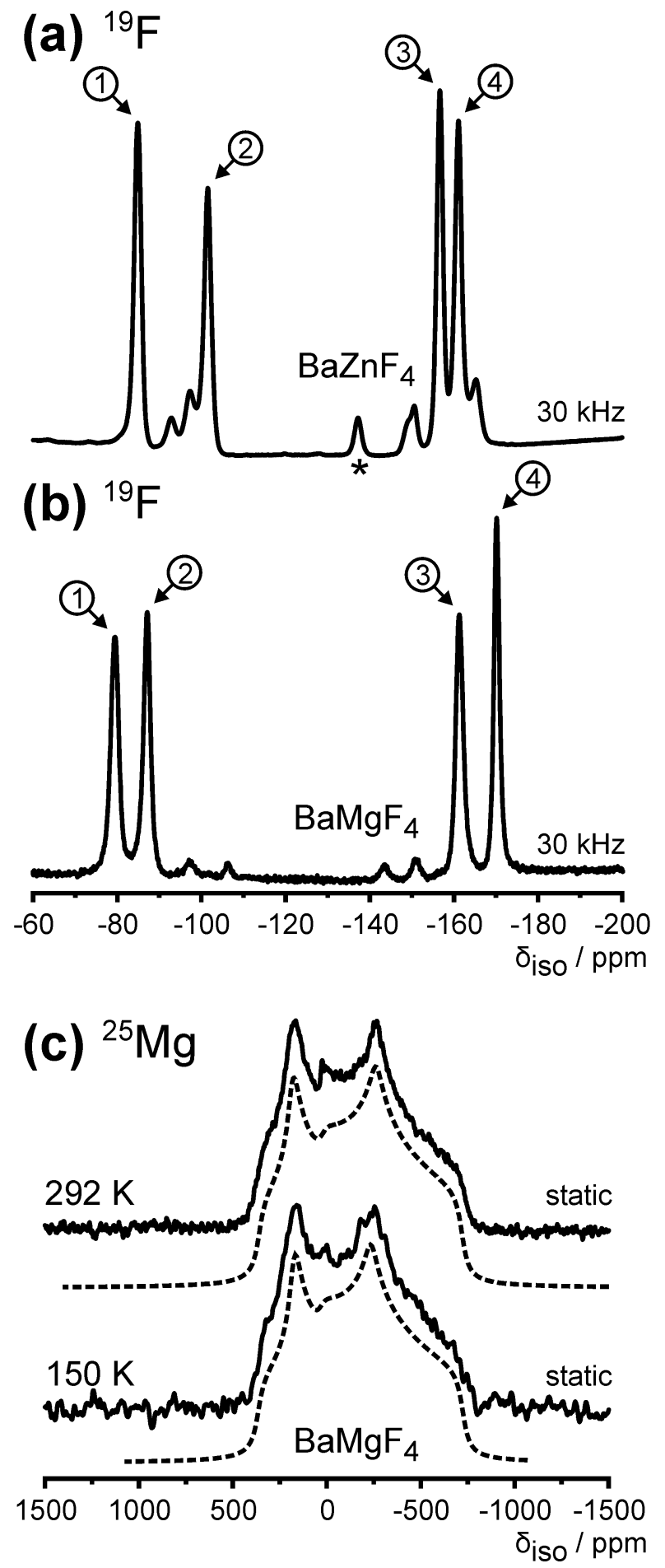

Figure 2. ${ }^{19} \mathrm{~F}$ solid-state MAS NMR spectra of $\mathrm{BaZnF}_{4}$ (a) and $\mathrm{BaMgF}_{4}$ (b) recorded at room temperature with observed peaks attributed to different fluorine nuclei in the unit cell (for their relation to the structure see figure 1). Asterisk denotes impurity; all other unnumbered peaks are spinning side bands. ${ }^{25} \mathrm{Mg}$ NMR spectra of $\mathrm{BaMgF}_{4}$ (c) recorded at $292 \mathrm{~K}$ and $150 \mathrm{~K}$ with their simulations (dashed lines). 
Table 1. Experimental and calculated (CASTEP [45]) ${ }^{19} \mathrm{~F}$ chemical shifts $\left(\delta_{\text {iso }}\right)$ for recorded NMR spectra of $\mathrm{BaZnF}_{4}$ and $\mathrm{BaMgF}_{4}$.

\begin{tabular}{llllll}
\hline & & $\delta_{\text {iso }}{ }^{\mathrm{F}(1)} / \mathrm{ppm}$ & $\delta_{\text {iso }}^{\mathrm{F}(2)} / \mathrm{ppm}$ & $\delta_{\text {iso }}{ }^{\mathrm{F}(3)} / \mathrm{ppm}$ & $\delta_{\text {iso }}^{\mathrm{F}(4)} / \mathrm{ppm}$ \\
\hline \multirow{2}{*}{$\mathrm{BaZnF}_{4}$} & Experiment & -84.8 & -101.5 & -156.6 & -161.0 \\
\cline { 2 - 6 } & Calculation & -101 & -104 & -160 & -181 \\
\hline \multirow{2}{*}{$\mathrm{BaMgF}_{4}$} & Experiment & -79.4 & -87.0 & -161.1 & -170.1 \\
\cline { 2 - 6 } & Calculation & -75 & -85 & -168 & -179 \\
\hline
\end{tabular}

(a)

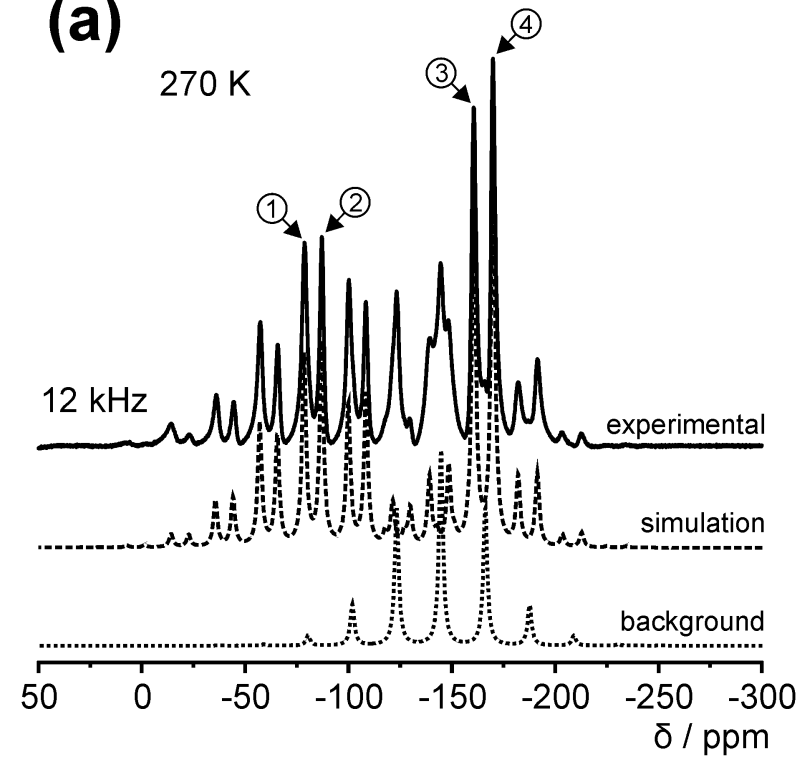

(a')

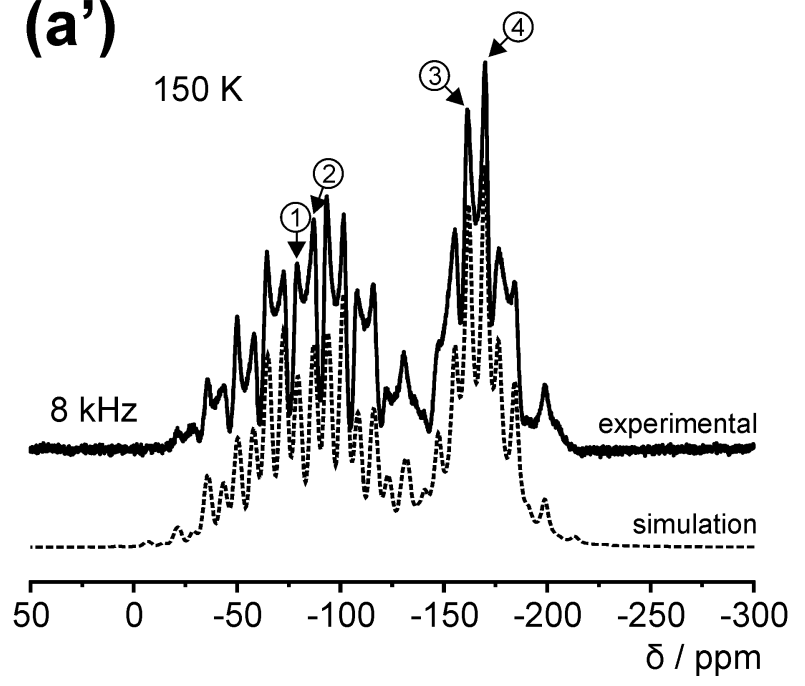

(b)
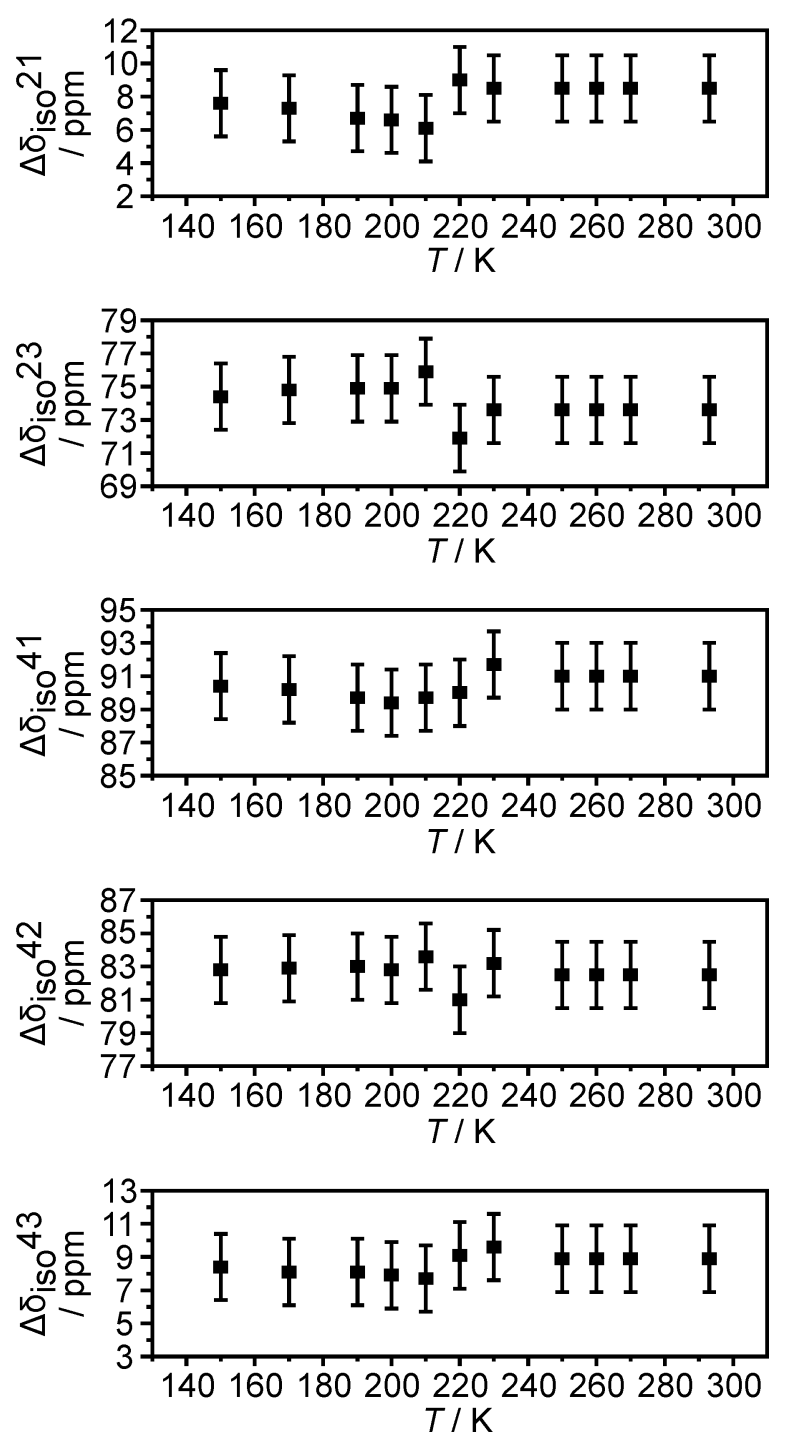

Figure 3. Variable temperature ${ }^{19} \mathrm{~F}$ solid-state MAS NMR spectra of $\mathrm{BaMgF}_{4}$ at $270 \mathrm{~K}$ (a) and $150 \mathrm{~K}$ (a'). The experimental spectra are given by solid lines, below are given simulated background-free spectra (dashed line) and simulated background components (dotted line). The $\delta_{\text {iso }}$ peaks are labelled in agreement with the structure scheme in figure 1. Temperature dependencies of relative differences in chemical shifts $\Delta \delta_{\text {iso }}{ }^{\mathrm{ij}}=\left|\delta_{\text {iso }}{ }^{\mathrm{F}(\mathrm{i})}-\delta_{\text {iso }}{ }^{\mathrm{F}(\mathrm{j})}\right|,(\mathrm{i}, \mathrm{j}=1, \ldots, 4)$ in the ${ }^{19} \mathrm{~F}$ MAS NMR spectrum of $\mathrm{BaMgF}_{4}$ (b). 
Representative variable temperature solid-state ${ }^{19} \mathrm{~F}$ MAS NMR spectra of $\mathrm{BaMgF}_{4}$ are shown in figure 3(a) and (a') (for $\mathrm{BaZnF}_{4}$ spectra see figure SM2 in Supplemental Material SM.1). They have complicated character which arises from the presence of four fluorine signals with significant Chemical Shift Anisotropies (CSA) and presence of strong background signals in the low temperature probe (see figure 3). However, application of the special procedure (described in details in Supplemental Material SM.1, figure SM1) allowed unambiguous identification and monitoring $\delta_{\text {iso }}$ with good precision ( $\pm 2 \mathrm{ppm})$ as function of temperature.

At each temperature point, all independent $\delta_{\text {iso }}$ (for each fluorine in unit cell), were found from the spectral simulation using SIMPSON [43]. For further analysis the differences in chemical shift (defined as $\Delta \delta_{\text {iso }}{ }^{i j}=\left|\delta_{\text {iso }}{ }^{F(i)}-\delta_{\text {iso }}{ }^{F(j)}\right|$, where the fluorine nucleus (i) is labelled $F(i)$ and $i, j=1, \ldots$, 4) were taken to eliminate any temperature shifts not related to the changes in the structure. The temperature dependencies of $\Delta \delta_{\text {iso }}^{\mathrm{ij}}$ for $\mathrm{BaMgF}_{4}$ is presented in figure 3(b), whereas for $\mathrm{BaZnF}_{4}$ in Supplemental Material figure SM1.

The values of $\Delta \delta_{\text {iso }}{ }^{i j}$ change only slightly over the temperature range $130-310 \mathrm{~K}$ as shown in figure 3(b) and figure SM2(b). This suggests that: (i) continuous deformation of the octahedron has only a very minor effect on $\delta_{\text {iso }}$ and (ii) its dependence on phonon dynamics can be neglected. In contrast to $\mathrm{BaZnF}_{4}$, for $\mathrm{BaMgF}_{4}$, the discontinuity in $\Delta \delta_{\text {iso }}^{\mathrm{ij}}$ at $\sim 210-220 \mathrm{~K}$ is observed (compare graphs in figure SM2(b) and figure 3(b)) and it is especially evident for $\Delta \delta_{\text {iso }}{ }^{21}$ and $\Delta \delta_{\text {iso }}{ }^{23}$ (figure 3(b)). These $\Delta \delta_{\text {iso }}{ }^{\mathrm{ij}}$ correspond to chemical shifts of fluorine nuclei that are lying at the octahedra corners in a plane parallel (or close to being parallel) to the $b c$ crystallographic plane, where octahedra form chains separated by $\mathrm{Ba}^{2+}$ nuclei (figure 1 ).

The origin of this discontinuity lays in the discontinuous change of the fluorine coordination sphere, which is directly related to the change of the octahedra geometry. The largest discontinuities observed for $\Delta \delta_{\text {iso }}{ }^{21}$ and $\Delta \delta_{\text {iso }}{ }^{23}$ (figure 3(b)), indicate that geometrical rearrangement takes place in the plane formed by $\mathrm{F}(1), \mathrm{F}(2)$ and $\mathrm{F}(3)$ fluorines (figure 1). Only very minor effects are observed when $\mathrm{F}(4)$ is involved. A similar temperature discontinuity in $\Delta \delta_{\text {iso }}{ }^{\mathrm{ij}}$ for $\mathrm{BaZnF}_{4}$ has not been detected because our variable temperature data have been restricted to the maximum achievable of only $300 \mathrm{~K}$ (figure SM2(b)).

Static broadline ${ }^{25} \mathrm{Mg}$ NMR spectra of $\mathrm{BaMgF}_{4}$ acquired at $14.1 \mathrm{~T}$ at temperatures of 292 and $150 \mathrm{~K}$ are presented in figure $2(\mathrm{c})$. The well-defined quadrupole lineshape describing the $(+1 / 2--1 / 2)$ central transition of the $I=5 / 2{ }^{25} \mathrm{Mg}$ nucleus is indicative of the highly crystalline lattice, and the short and long range positional order influencing the magnesium environment [49]. At room temperature spectral parameters ( $\delta_{\text {iso }} \approx 0 \mathrm{ppm}, \mathrm{C}_{\mathrm{q}} \approx 5.7 \mathrm{MHz}$ and $\eta \approx 0.47$ ) are in the range typical for distorted octahedral coordination environment as reported for number of studied organic and inorganic compounds $[50,51]$. There exists some discrepancy between the GIPAW DFT calculated 


\section{Molecular dynamics in geometric ferroelectric fluorides}

quadrupole parameters obtained using the CASTEP code $\left(\mathrm{C}_{\mathrm{q}}=7.7 \mathrm{MHz}, \eta=0.51\right)$ and the measured values reported above, although previous studies comparing calculated and measured ${ }^{25} \mathrm{Mg} \mathrm{NMR}$ parameters have reported similar discrepancies for systems characterised by larger field gradients $\left(\mathrm{C}_{\mathrm{q}}\right.$ values) [51]. This observation is ascribed to the limitations of the pseudopotentials used in these calculations.

No significant changes in spectral parameters have been observed between 292 and $150 \mathrm{~K}$ (figure 2(c)), despite the potential of ${ }^{25} \mathrm{Mg}$ to be a very sensitive probe of the local environment through the changes in quadrupolar interaction [51]. However, such "insensitivity" cannot provide definitive evidence of the high structural stability because the observed broad line spectrum could mask very subtle changes in ${ }^{25} \mathrm{Mg}$ coordination. Additionally, only more detailed and systematic studies over a significantly broader range of temperatures can bring definitive confirmation of such "insensitivity". 


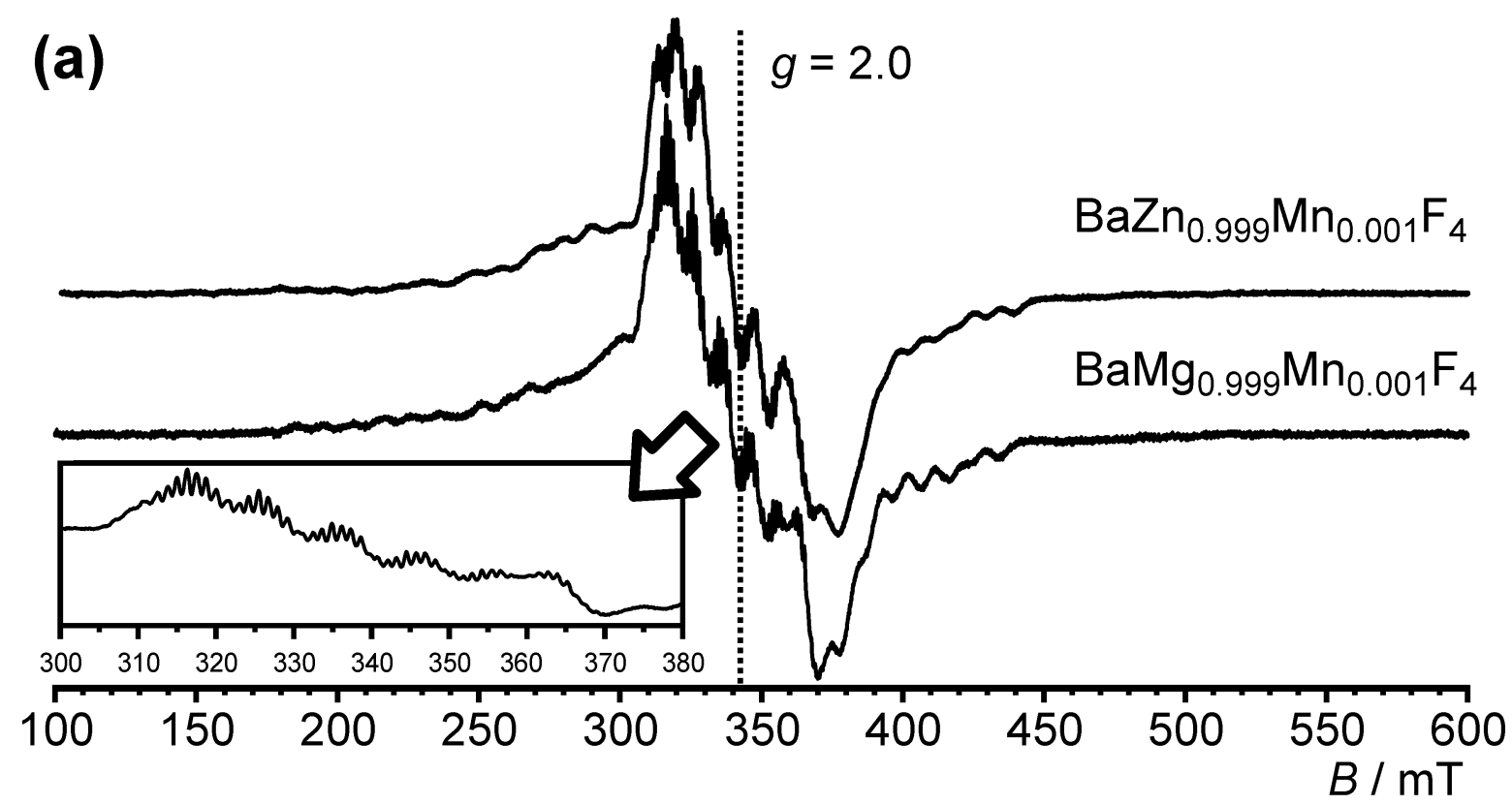

(b) $\mathrm{BaZn}_{0.999} \mathrm{Mn}_{0.001} \mathrm{~F}_{4}$

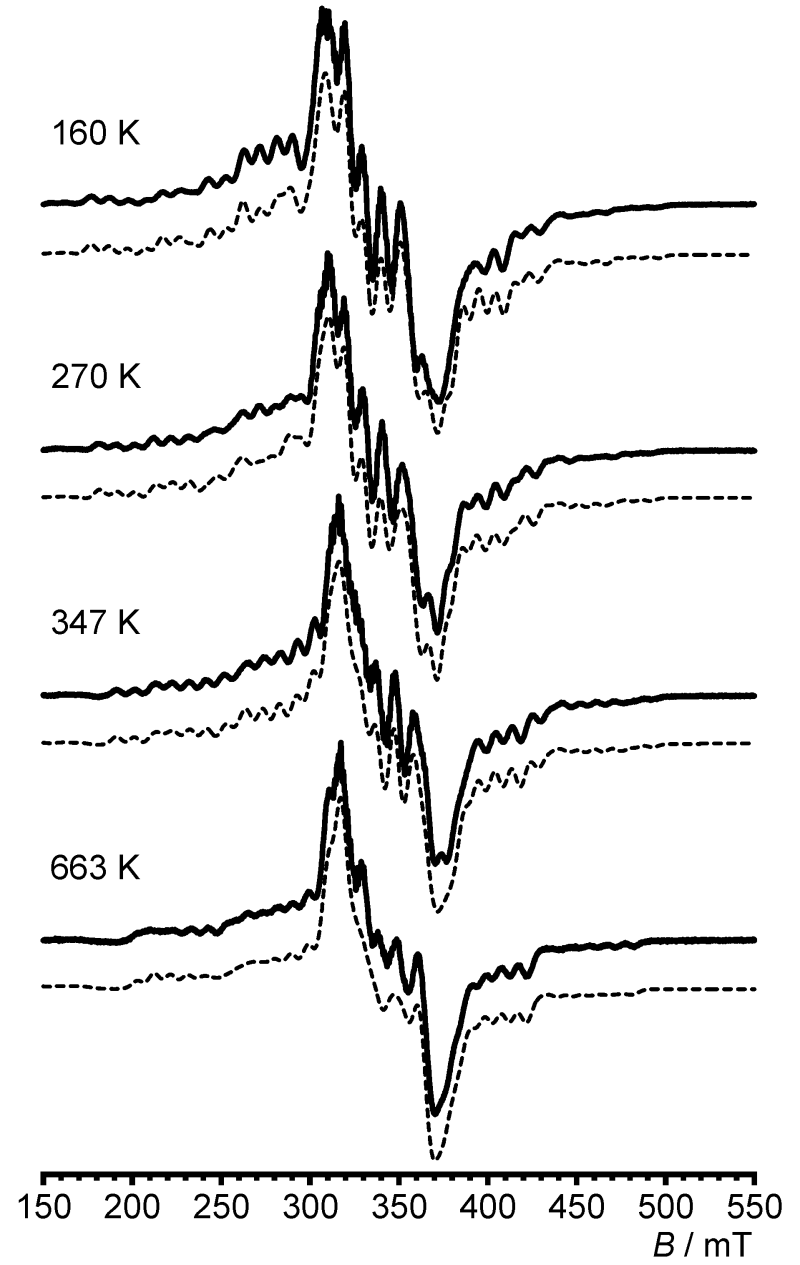

(c) $\mathrm{BaMg}_{0.995} \mathrm{Mn}_{0.005} \mathrm{~F}_{4}$

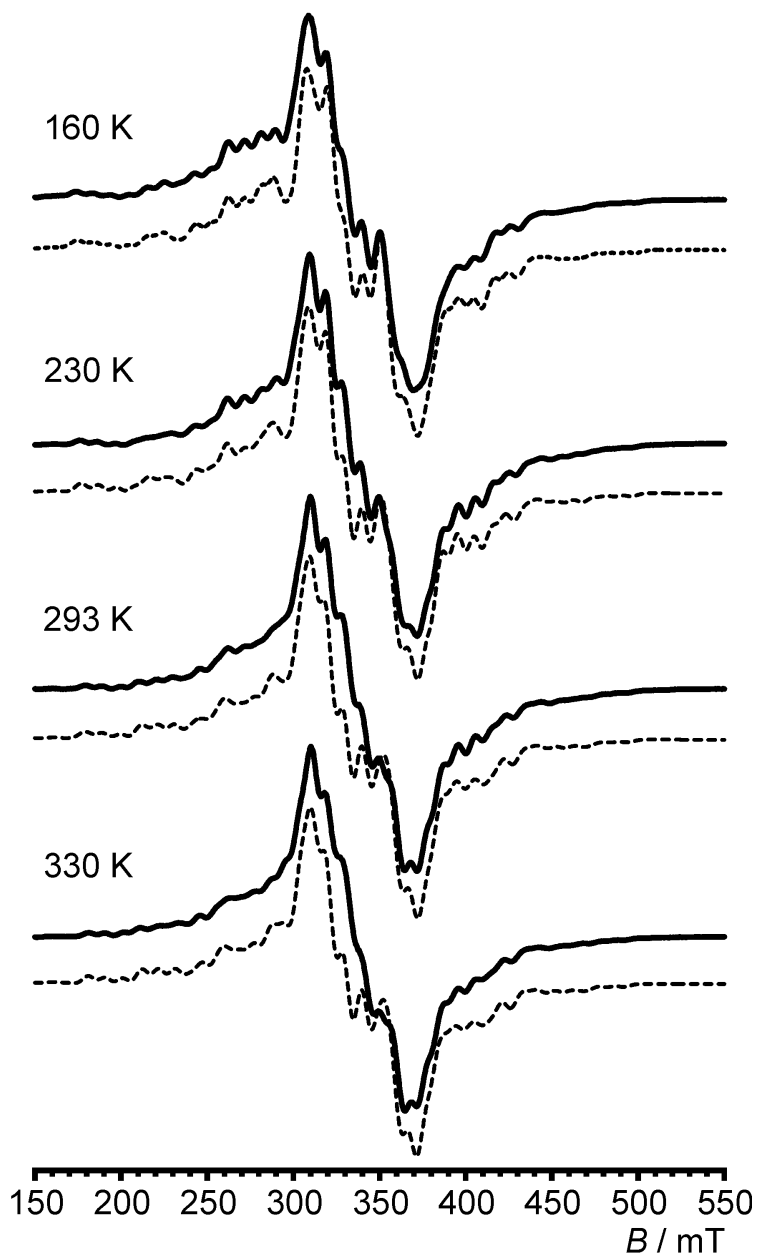

Figure 4. Room temperature $X$-band $E P R$ spectra of $B a{ }_{0.999} \mathrm{Mn}_{0.001} \mathrm{~F}_{4}$ and $\mathrm{BaMg}_{0.991} \mathrm{Mn}_{0.001} \mathrm{~F}_{4}$ (a) with an inset showing the central part of the spectrum with superhyperfine structure. Variable temperature X-band EPR spectra and their spectral simulations (dashed line) of $\mathrm{BaZn}_{0.999} \mathrm{Mn}_{0.001} \mathrm{~F}_{4}$ (b) and $\mathrm{BaMg}_{0.995} \mathrm{Mn}_{0.005} \mathrm{~F}_{4}(\mathrm{c})$. 


\subsection{Electron Paramagnetic Resonance (EPR)}

The X-band (9.450 GHz) EPR spectra of paramagnetic $\mathrm{Mn}^{2+}(\mathrm{S}=5 / 2, \mathrm{I}=5 / 2)$ doped $(0.1 \%)$ into a diamagnetic matrix of $\mathrm{BaZnF}_{4}$ and $\mathrm{BaMgF}_{4}$ are presented in figure 4(a). Remarkably well resolved structures of 30 resonances in the spectrum (five, partially overlapped, groups of six lines assigned to allowed transition) suggests a high level of order in the crystal structure surrounding the doped ion, which replaces the $\mathrm{Mg}^{2+}$ or $\mathrm{Zn}^{2+}$ in the dielectric matrix and has a well defined microenvironment. The central transition $(-1 / 2 \rightarrow+1 / 2)$, at $g \sim 2.00$ is split further due to a superhyperfine interaction of the unpaired electron with six fluorine nuclei $(I=1 / 2)$ located at the corners of fluoride octahedra (see inset in figure 4(a) and figure 1). For other transitions this splitting is not observed due to line broadening related to ZFS.

For the higher doping levels the remarkably well resolved superhyperfine structure disappears due to the increase of linewidth caused by dipole - dipole interactions (for details see Supplemental Material SM.2, figure SM3). Such behaviours confirms the presence of the strong long-range interaction between distant $\mathrm{Mn}^{2+}$ ions.

The hyperfine constant for $\mathrm{Mn}^{2+}(\mathrm{I}=5 / 2)$ found from spectral simulations is $A^{M n}$ iso $\approx 270$ $\mathrm{MHz}$ and the hyperfine constants for $\mathrm{F}^{-}(\mathrm{I}=1 / 2)$ are $A_{\text {iso }}^{F} \approx 38$ and $34 \mathrm{MHz}$ for $\mathrm{BaZn}_{0.999} \mathrm{Mn}_{0.001} \mathrm{~F}_{4}$ and $\mathrm{BaMg}_{0.999} \mathrm{Mn}_{0.001} \mathrm{~F}_{4}$, respectively at room temperature. The value of the hyperfine splitting constant is in the range typical for $\mathrm{Mn}^{2+}$ ions [52] and depends very weakly on temperature. The small hyperfine value $\mathrm{F}^{-}$indicates a low level of delocalisation on the fluorines.

For $\mathrm{BaZn}_{1-\mathrm{x}} \mathrm{Mn}_{\mathrm{x}} \mathrm{F}_{4}(\mathrm{x}=0.001$ and 0.005$)$, the estimated ZFS parameters are $|D| \sim 0.03 \mathrm{~cm}^{-1}$ (906 MHz) and $|E| \sim 0.006 \mathrm{~cm}^{-1}(188 \mathrm{MHz})$ at room temperature. For $\mathrm{BaMg}_{1-\mathrm{x}} \mathrm{Mn}_{\mathrm{x}} \mathrm{F}_{4}(\mathrm{x}=0.001$ and $0.005)$, ZFS parameters are $|D| \sim 0.03 \mathrm{~cm}^{-1}(930 \mathrm{MHz})$ and $|E| \sim 0.006 \mathrm{~cm}^{-1}(176 \mathrm{MHz})$ in agreement with previous single-crystal studies [33]. The ZFS parameters $|D|$ and $|E|$ provide a reliable measure of the strength of the crystal field in both matrices $\mathrm{BaZnF}_{4}$ and $\mathrm{BaMgF}_{4}$. The relatively large value of Zero Field Splitting parameter $|D|$ supports the observation that most of the electron spin density is localized on the paramagnetic ion surrounded by distorted octahedra. The ZFS parameter $|E|$ permits an assessment of the significant distortion of the crystal field, which changes from distorted axial symmetry at low temperatures to nearly rhombic at high temperatures (figure 4 and figure 5).

Temperature evolution of $\mathrm{BaZn}_{0.999} \mathrm{Mn}_{0.001} \mathrm{~F}_{4}$ and $\mathrm{BaMg}_{0.995} \mathrm{Mn}_{0.005} \mathrm{~F}_{4}$ spectra, together with their spectral simulations, are presented, in figure 4(b) and 4(c). The pattern of resonances reflects allowed transitions in the spin manifolds. Changes in the linewidth, intensities and ZFS are responsible for differences between low and high temperature spectra. The high resolution of X-band spectra as well as good agreement between experiment and simulation allowed precise estimation of ZFS parameters $|D|$ and $|E|$. It was also possible to restrict EPR experiments to X-band frequencies 
where more details of the spectral pattern are resolved compared to Q-band spectra (for details see Supplemental Material SM.3, figure SM4).
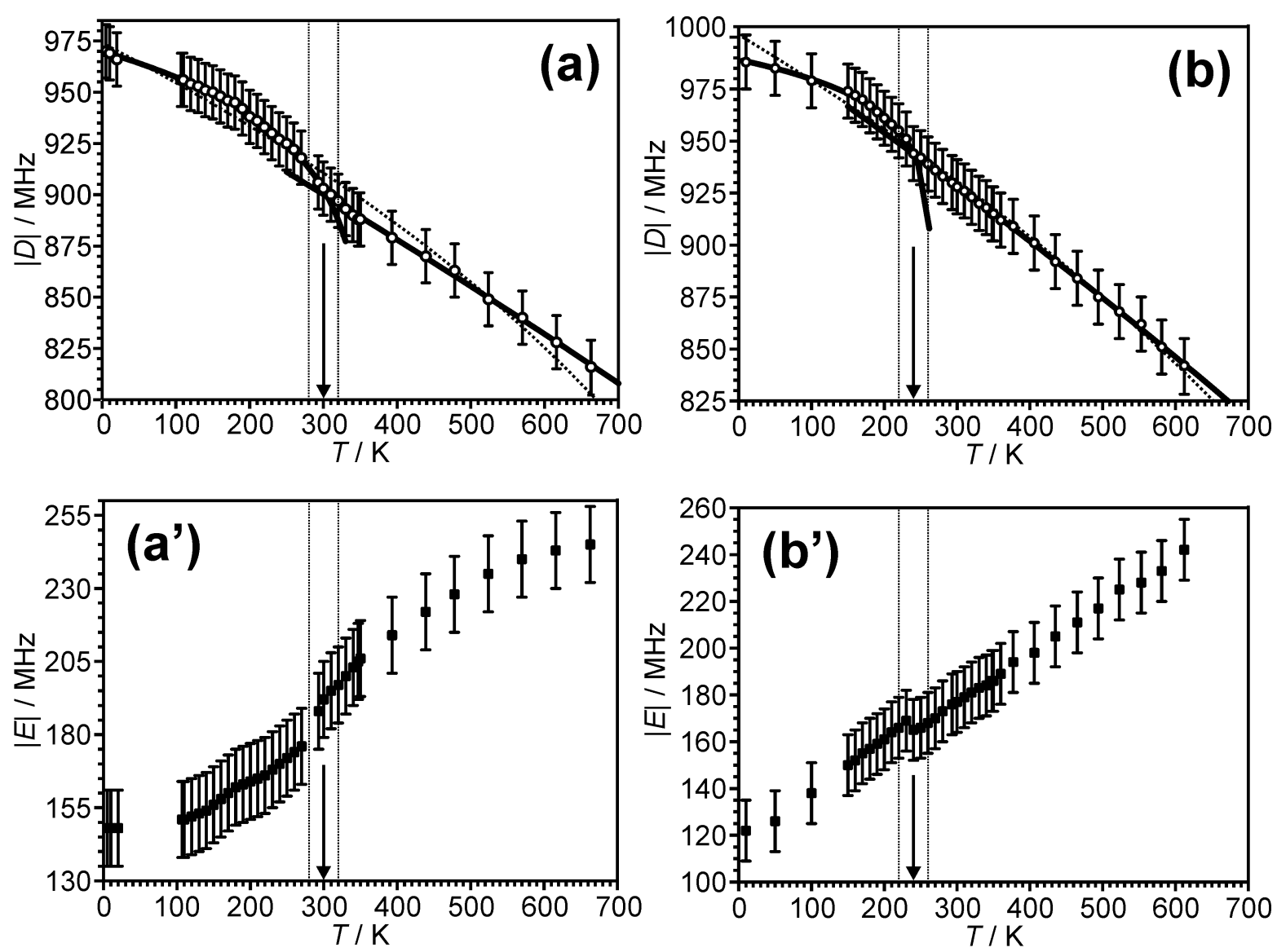

Figure 5. Temperature dependence of ZFS parameters $|D|$ (a), (b) and $|E|$ (a'), (b') for $\mathrm{BaZn}_{0.999} \mathrm{Mn}_{0.001} \mathrm{~F}_{4}$ (a) and (a') and $\mathrm{BaMg}_{0.995} \mathrm{Mn}_{0.005} \mathrm{~F}_{4}$ (b) and (b'). The $\mathrm{Y} \propto\left(\mathrm{A}_{\mathrm{C}}-\mathrm{X}\right)^{\mathrm{B}}$ formula was used to fit the $|D|$ vs. T curve in the whole temperature range (dotted line), in the low temperature (5 $240 \mathrm{~K})$ region (solid line) and high temperature $(250$ - $650 \mathrm{~K})$ region (solid line). Vertical dotted lines indicate the temperature range at which discontinuity occurs.

Temperature dependencies of $|D|$ and $|E|$ obtained from the spectral simulations are presented in figure 5. For both samples as temperature increases, $|D|$ decreases and $|E|$ increases. These temperature dependencies are governed by two factors: static - related to the structural changes reflected in thermal expansion, and dynamic - related to strength of the coupling between spin and phonons $[29,53]$. In the case of fluorides both contributions are expected to be present and equally important, because the continuous structural distortions are strongly related to both thermal expansion and dynamics of phonons. The shape of the temperature dependencies of ZFS is influenced by both factors. A detailed discussion of the temperature behaviour of ZFS, in relation to molecular dynamics is given in the next section. 


\section{Molecular dynamics in geometric ferroelectric fluorides}

The $\mathrm{Y} \propto\left(\mathrm{A}_{\mathrm{C}}-\mathrm{X}\right)^{\mathrm{B}}$ formula (analogous to the equation describing the order parameter as a function of temperature in the ferroelectric phase) was used to fit $|D|$ vs. $T$ curves in the studied temperature range (figure 5(a) and 5(b)). The fitting curves obtained, despite lying in the range of experimental errors do not sufficiently reflect the course of the dependency, which is especially evident for $\mathrm{BaZn}_{0.999} \mathrm{Mn}_{0.001} \mathrm{~F}_{4} \cdot|D|$ vs. $T$ curves show subtle (at the limits of experimental errors) changes in the course of dependency from close to linear at higher temperature to non-linear at lower temperatures (figure 5(a) and 5(b)). Using the same fitting formula $\left(\mathrm{Y} \propto\left(\mathrm{A}_{\mathrm{C}}-\mathrm{X}\right)^{\mathrm{B}}\right)$ separately for the low (see figure 5 caption for details) and high temperature ranges significantly improves agreement and provides two distinct characters of the dependency. For $\mathrm{BaZn}_{0.999} \mathrm{Mn}_{0.001} \mathrm{~F}_{4}$ the estimated temperature at which the change occurs is $\sim 300 \pm 20 \mathrm{~K}$. For $\mathrm{BaMg}_{0.995} \mathrm{Mn}_{0.005} \mathrm{~F}_{4}$ the temperature at which the change occurs is at $\sim 240 \pm 20 \mathrm{~K}$. Correspondingly, anomalies in the temperature dependency of $|E|$ occur, at $\sim 300 \pm 20 \mathrm{~K}$ for $\mathrm{BaZn}_{0.999} \mathrm{Mn}_{0.001} \mathrm{~F}_{4}$ and $\sim 240 \pm 20 \mathrm{~K}$ for $\mathrm{BaMg}_{0.995} \mathrm{Mn}_{0.005} \mathrm{~F}_{4}$ (figure 5(a') and (b')) which are in very good agreement with the change in character of $|D|$. The most likely mechanism of those changes is related to discontinuous changes of the geometry of the octahedra presented in detail in the next section.

The $g$ factor and hyperfine interaction $A^{M n}$ iso depend very weakly on temperature and change in the range of experimental errors.

\subsection{Raman scattering}

Depolarized Raman spectra recorded at room temperature for all investigated samples are presented in figure 6. For group symmetry $\mathrm{C}_{2 \mathrm{v}}{ }^{12}$ and 4 molecules in the unit cell 33 Raman active modes are expected from group theory in the ferroelectric phase of $\mathrm{BaMF}_{4}\left(\mathrm{M}^{2+}=\mathrm{Mg}^{2+}, \mathrm{Zn}^{2+}, \mathrm{Co}^{2+}, \mathrm{Fe}^{2+}, \mathrm{Ni}^{2+}\right.$, $\left.\mathrm{Mn}^{2+}\right): 11 \mathrm{~A}_{1}+6 \mathrm{~A}_{2}+5 \mathrm{~B}_{1}+11 \mathrm{~B}_{2}[25,33,54]$. In our experiments the total number of observed bands for all investigated materials does not exceed 13, a situation which is not unusual considering band overlap, low intensity bands and our spectral cut-off at $80 \mathrm{~cm}^{-1}$. Even though, the symmetry assignment of the bands is difficult because of the depolarized nature of the spectra. However, all observed bands are in good agreement with previously published data (for details see Supplemental Material SM.4, table SM1) [34, 37]. 

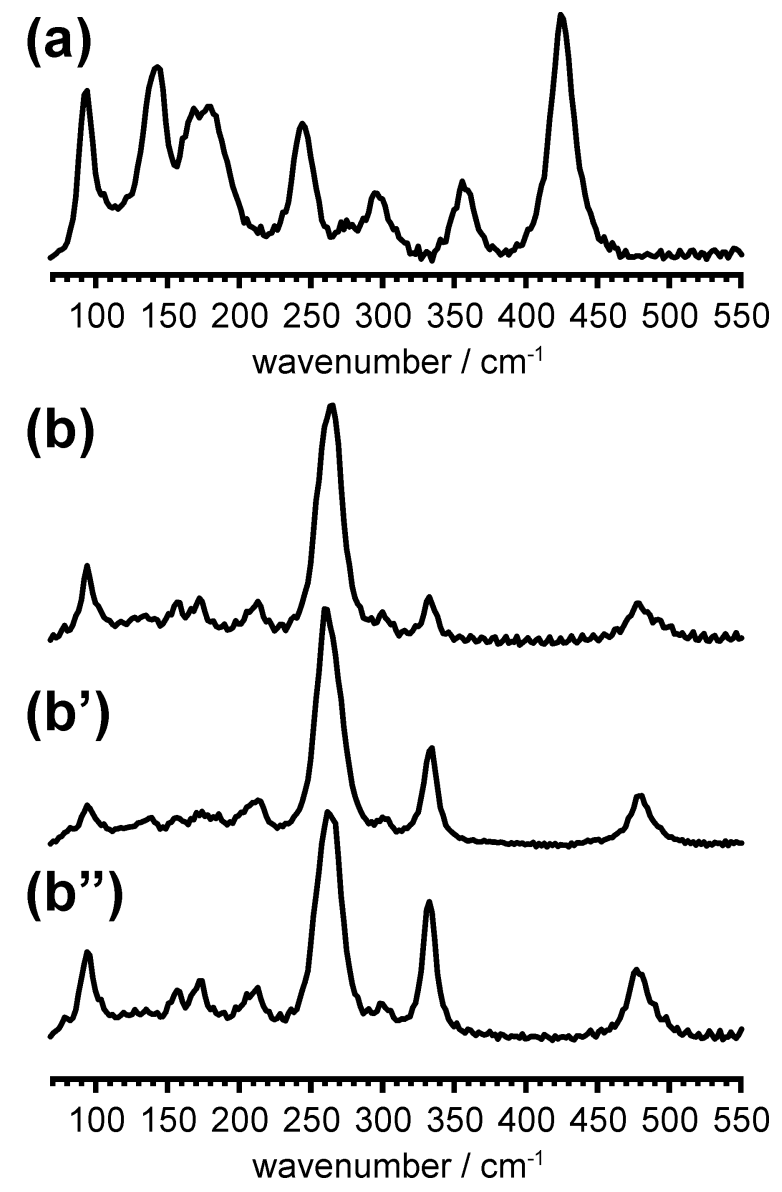

Figure 6. Depolarized Raman spectra of $\mathrm{BaZnF}_{4}$ (a), $\mathrm{BaMgF}_{4}$ (b), $\mathrm{BaMg}_{0.999} \mathrm{Mn}_{0.001} \mathrm{~F}_{4}$ (b') and $\mathrm{BaMg}_{0.995} \mathrm{Ni}_{0.005} \mathrm{~F}_{4}$ (b') recorded at room temperature for randomly oriented crystals.

The observed phonon spectrum is a spectral signature of the structure of the studied fluorides and it is expected that the room temperature Raman spectra will have a similar pattern of bands for all members of the family, as well as for samples modified by doping, in agreement with our observation [34]. However, when comparing $\mathrm{BaMgF}_{4}$, and low level doped $\mathrm{BaMg}_{0.999} \mathrm{Mn}_{0.001} \mathrm{~F}_{4}$ and $\mathrm{BaMg}_{0.995} \mathrm{Ni}_{0.005} \mathrm{~F}_{4}$ it can be seen that frequencies of some bands are affected by the chemical composition (see table SM1 in Supplemental Material), as earlier observed for $\mathrm{Mn}^{2+}$ doped $\mathrm{BaZnF}_{4}$ [28]. For instance, the bands at $\sim 258 \mathrm{~cm}^{-1}, 266 \mathrm{~cm}^{-1}$ and $333 \mathrm{~cm}^{-1}$ in $\mathrm{BaMgF}_{4}$ shift to higher frequencies in $\mathrm{BaMg}_{0.999} \mathrm{Mn}_{0.001} \mathrm{~F}_{4}$ (by approximately 0.3 to $2 \mathrm{~cm}^{-1}$ ), but to lower frequency in $\mathrm{BaMg}_{0.995} \mathrm{Ni}_{0.005} \mathrm{~F}_{4}$.

Such a consistent trend for phonon frequencies in the doped samples is related to the level of structural distortion (which is induced by a large ionic radius) rather than the difference in the mass of the dopant as observed previously in [28]. This model is also consistent with our observation, that even at very small levels of doping $(0.1 \%)$ the shifts are dominated by the level of distortion due to differences between ionic radius $\mathrm{Mg}^{2+}(\mathrm{r}=0.66 \AA)<\mathrm{Zn}^{2+}(\mathrm{r}=0.74 \AA)<\mathrm{Mn}^{2+}(\mathrm{r}=0.84 \AA)$ rather than differences in the mass $\left[\mathrm{Mg}^{2+}(\mathrm{m}=23.98)<\mathrm{Mn}^{2+}(\mathrm{m}=54.93)<\mathrm{Zn}^{2+}(\mathrm{m}=63.92)\right]$. 
Some small differences in the amplitudes of less intense bands between 150 and $190 \mathrm{~cm}^{-1}$ are observed, probably due to small differences in the orientation of the crystals between investigated samples.

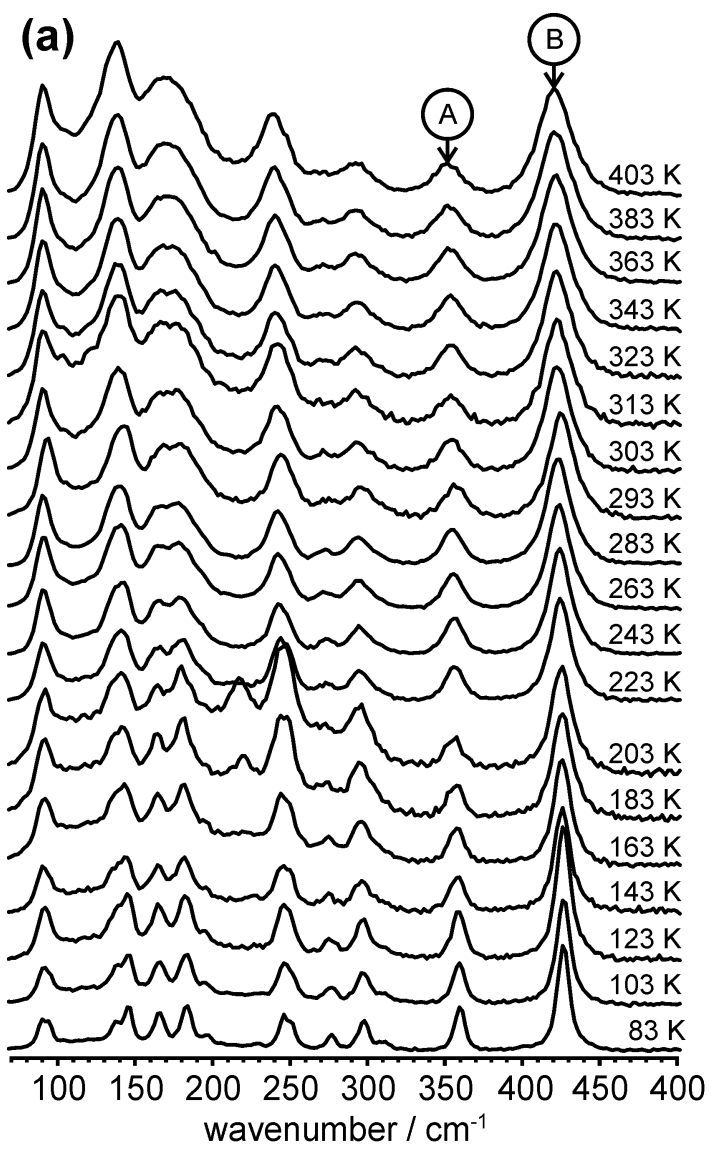

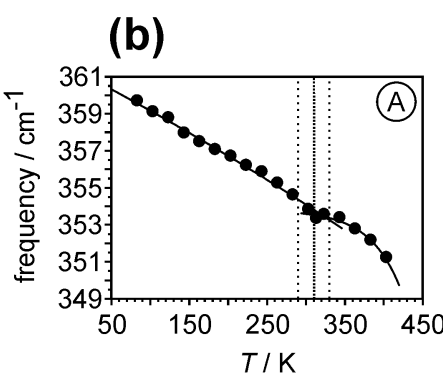

(b')

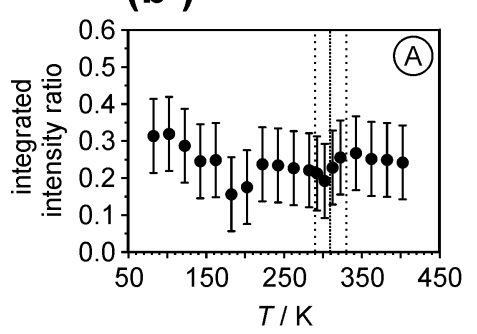

(b")

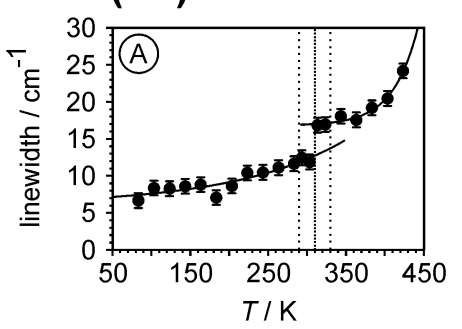

(c)

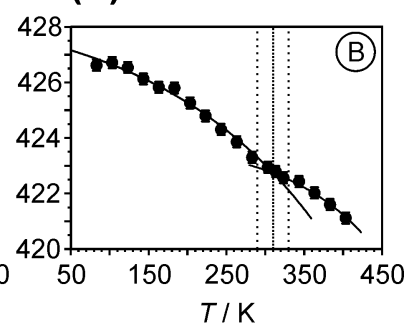

(c')

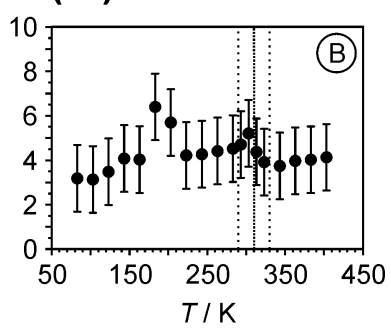

(c")

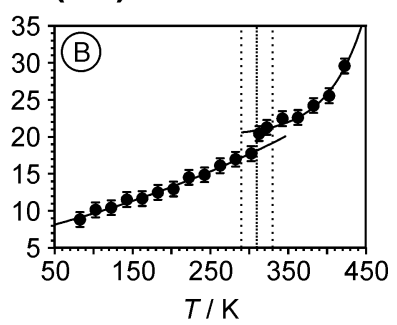

Figure 7. Variable temperature Raman spectra of $\mathrm{BaZnF}_{4}$ (a). For the clarity all spectra were normalised in reference to the amplitude of band A recorded at $83 \mathrm{~K}$. Temperature changes of the frequency (b) and (c), integrated intensity ratios (b') and (c') and linewidths (b") and (c') for selected bands A and B. Solid lines are guides for the eye only. Vertical dotted lines indicate temperature range in which the discontinuity occurs (marked by bold dotted line).

Temperature-dependent Raman spectra recorded for $\mathrm{BaZnF}_{4}$ are presented in figure 7(a). The low temperature spectrum $(83 \mathrm{~K})$ is characterized by sharp bands with the most intense one at $\sim 425$ $\mathrm{cm}^{-1}$. With increasing temperature the expected trend of linewidth broadening and shift towards lower frequencies is observed which results in an increased band overlap. The exception is bands at $\sim 360$ $\mathrm{cm}^{-1}$ (labelled A in figure 7(a)) and $\sim 425 \mathrm{~cm}^{-1}$ (labelled B in figure 7(a)) which remain isolated over the whole temperature range. Such Raman bands at high-frequencies are considered as particularly sensitive to small structural changes [28].

The temperature evolution of the single-line bands A and B is presented in figure 7, by comparing the evolution in positions (figure 7(b) and (c)), integrated intensity ratios (figure 7(b') and $\left(c^{\prime}\right)$ ) and linewidths (figure $7\left(b^{\prime \prime}\right)$ and $\left.\left(c^{\prime \prime}\right)\right)$. As seen from figure 7 , the spectral changes are very 
subtle and indicate that no major structural transition takes place. Nevertheless, we note slight anomalies at around $310 \mathrm{~K}$ which might indicate a local and subtle structural rearrangement related to changes in molecular dynamics. It is necessary to stress that, those anomalies occur at the temperature range for which we earlier observed changes in ZFS parameters. Earlier papers [25-28] also report spectral changes at approximately $170 \mathrm{~K}$ for low frequency bands in the $\mathrm{BaZnF}_{4}$ spectrum, but the very subtle changes in our spectra at the same temperature are not evidence of themselves for any structural change.

(a)

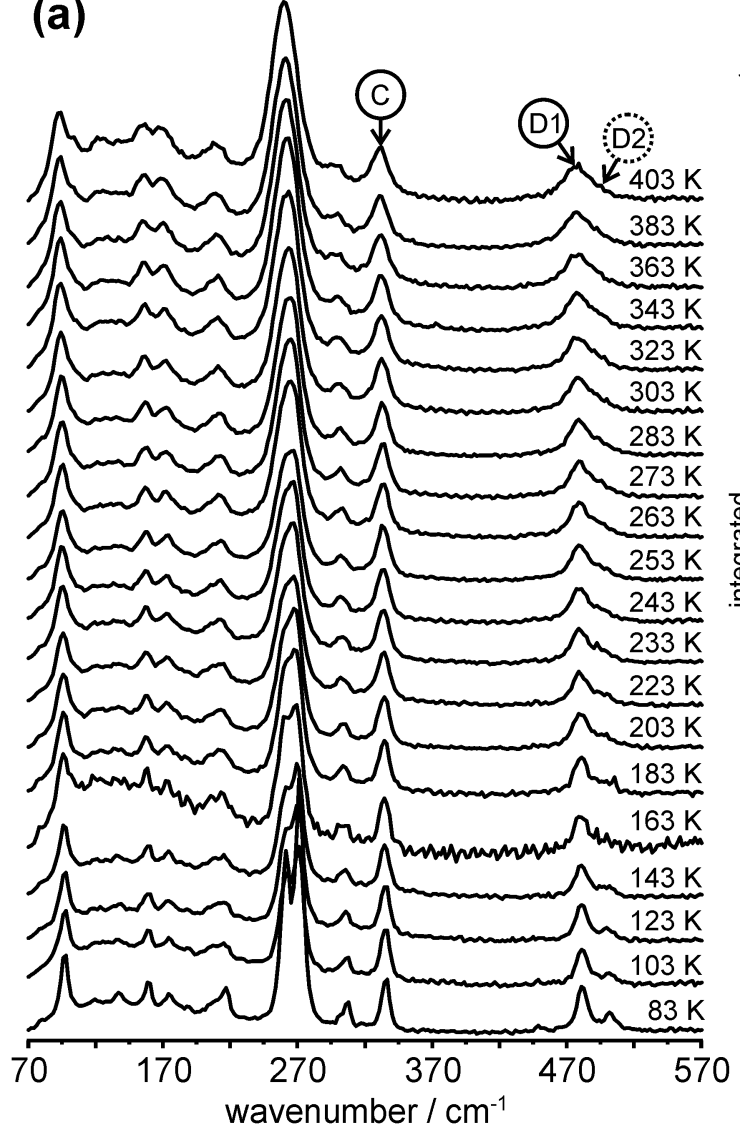

(b)

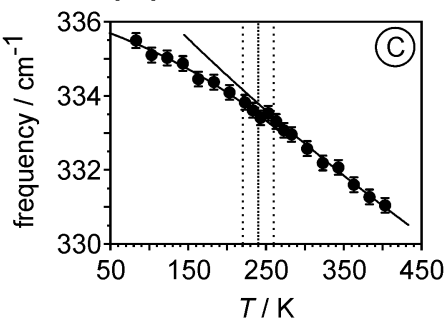

(b')

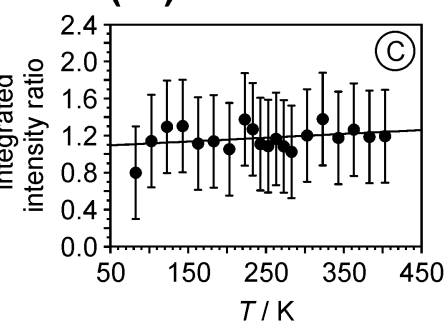

(b")

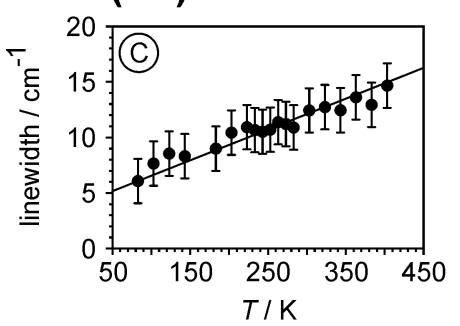

(c)

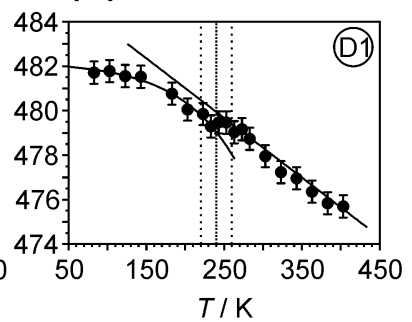

(c')

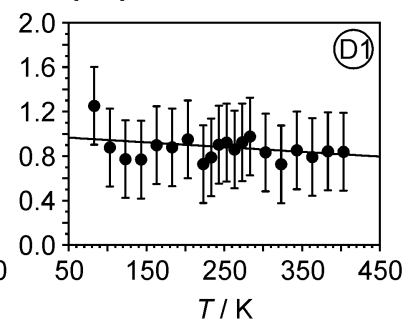

(c")

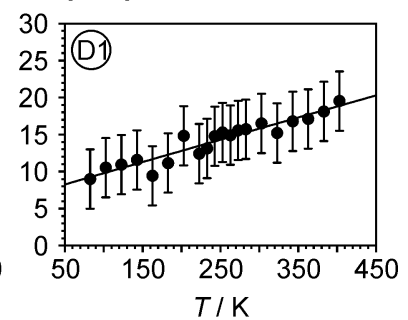

Figure 8. Variable temperature Raman spectra of $\mathrm{BaMgF}_{4}$ (a). For the clarity all spectra were normalised in reference to the amplitude of band $\mathrm{C}$ recorded at $83 \mathrm{~K}$. Temperature changes of the frequency (b) and (c), integrated intensity ratios (b') and (c') and linewidths (b') and (c'") for selected bands C and D1. Solid lines are guides for the eye only. Vertical dotted lines indicate temperature range in which the discontinuity occurs (marked by bold dotted line). The temperature dependency of spectral characteristic for band C is given in Supplemental material.

Figures 8(a), 9(a) and 10(a) present, to the best of our knowledge, the first temperaturedependent Raman spectra of $\mathrm{BaMgF}_{4}, \mathrm{BaMg}_{0.999} \mathrm{Mn}_{0.001} \mathrm{~F}_{4}$ and $\mathrm{BaMg}_{0.995} \mathrm{Ni}_{0.005} \mathrm{~F}_{4}$, respectively in the range of $83 \mathrm{~K}$ to $403 \mathrm{~K}$. The temperature-dependent spectra of $\mathrm{BaMgF}_{4}$ and $\mathrm{BaMg}_{0.999} \mathrm{Mn}_{0.001} \mathrm{~F}_{4}$ display a very similar structure. All spectra are well defined at low temperature with an intense doublet at $\sim 270$ and $\sim 280 \mathrm{~cm}^{-1}$ which merges into a single band due to thermal broadening upon 
heating (figures 8(a), 9(a)). The pattern of bands in the Raman spectrum of $\mathrm{BaMg}_{0.995} \mathrm{Ni}_{0.005} \mathrm{~F}_{4}$ resembles that of $\mathrm{BaMgF}_{4}$ and $\mathrm{BaMg}_{0.999} \mathrm{Mn}_{0.001} \mathrm{~F}_{4}$, with a dominating central doublet. However, some differences in the pattern of less intense bands are observed, probably due to small differences in the orientation of the crystals between samples.

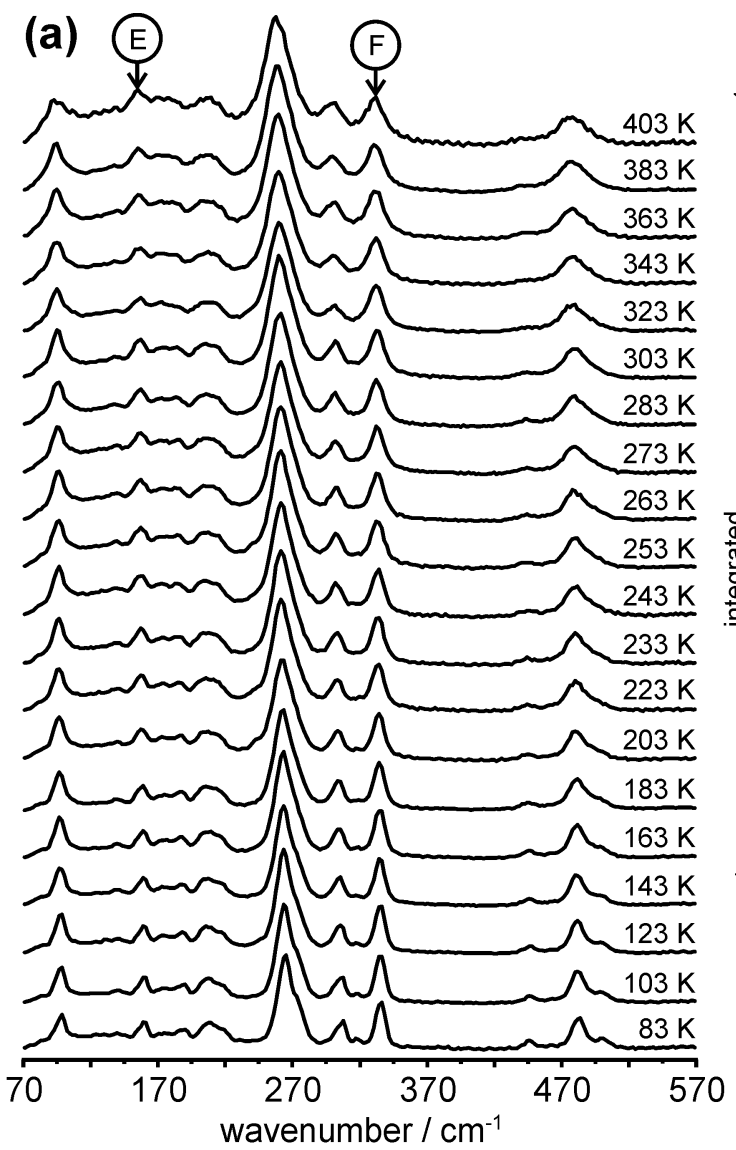

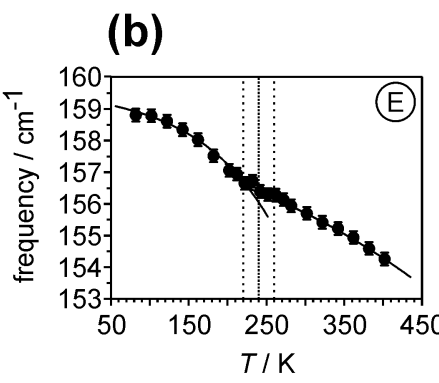

(b')

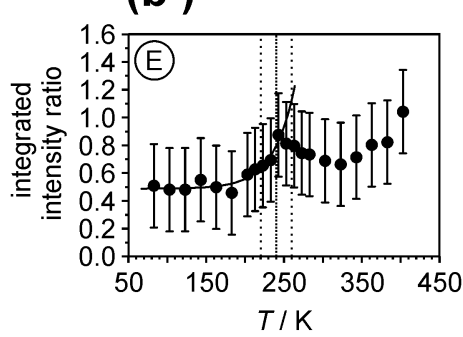

(b")

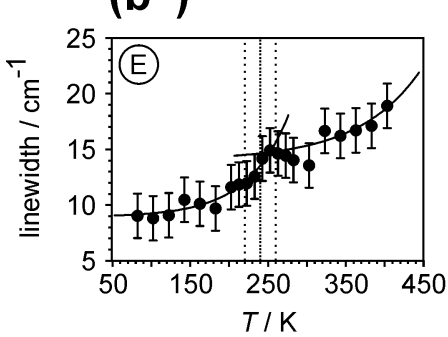

(c)

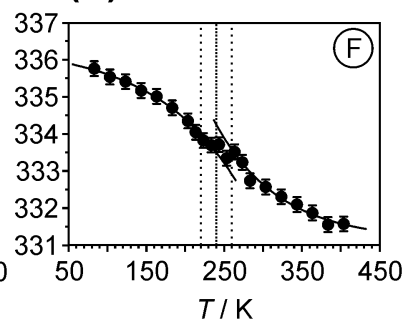

(c')

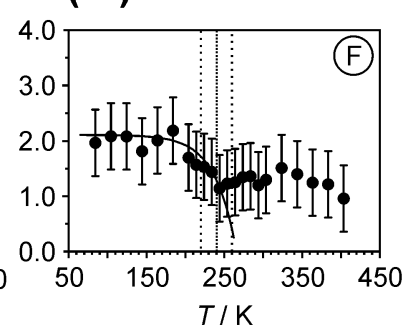

(c")

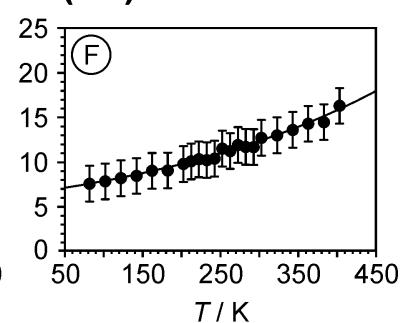

Figure 9. Variable temperature Raman spectra of $\mathrm{BaMg}_{0.999} \mathrm{Mn}_{0.001} \mathrm{~F}_{4}$ (a). For the clarity all spectra were normalised in reference to the amplitude of band $\mathrm{F}$ recorded at $83 \mathrm{~K}$. Temperature changes of the frequency (b) and (c), integrated intensity ratios (b') and (c') and linewidths (b') and (c") for selected bands E and F. Solid lines are guides for the eye only. Vertical dotted lines indicate temperature range in which the discontinuity occurs (marked by bold dotted line). 


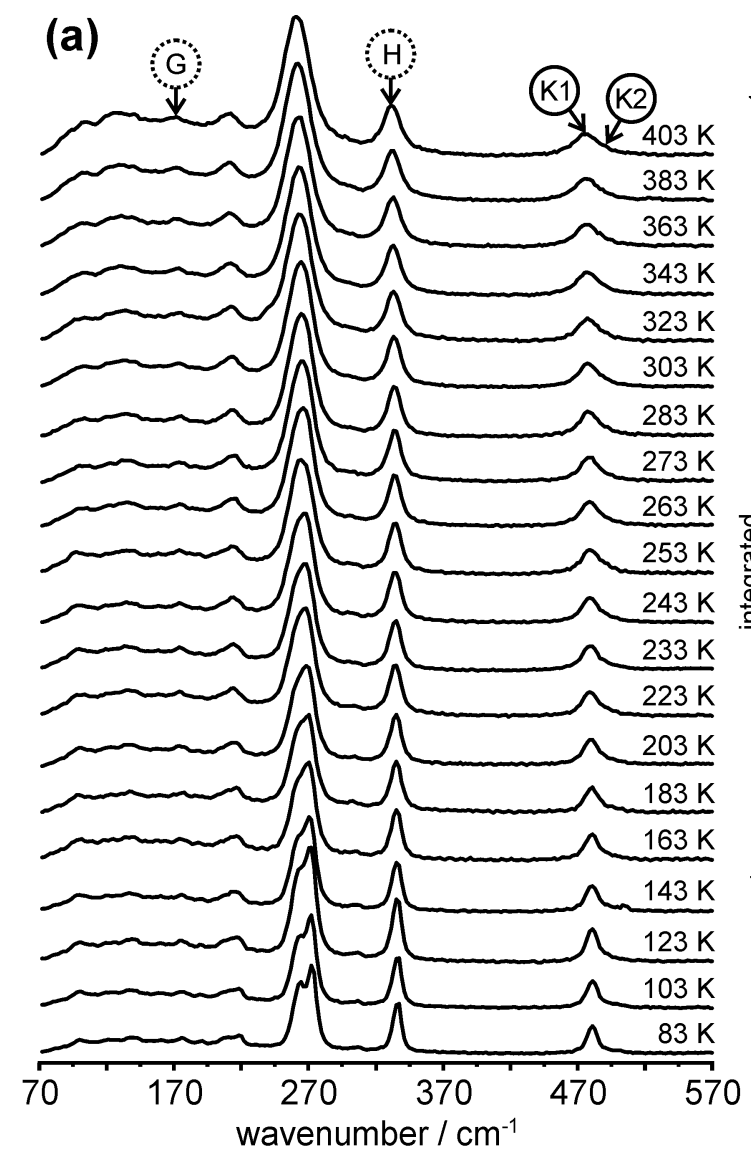

(b)

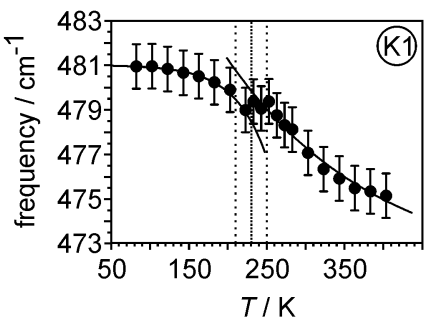

(b')

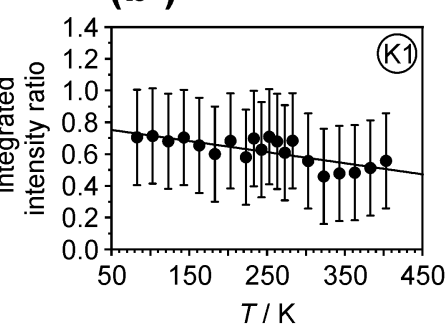

(b")

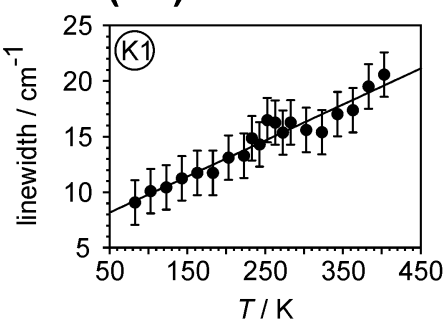

(c)

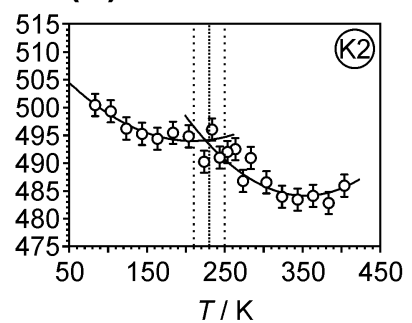

(c')

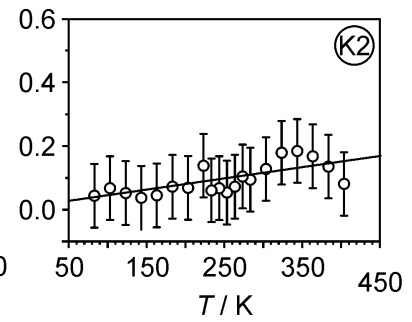

(c")

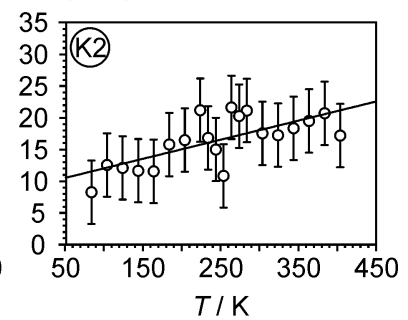

Figure 10. Variable temperature Raman spectra of $\mathrm{BaMg}_{0.995} \mathrm{Ni}_{0.005} \mathrm{~F}_{4}$ (a). For the clarity all spectra were normalised in reference to the amplitude of band $\mathrm{H}$ recorded at $83 \mathrm{~K}$. Temperature changes of the frequency (b) and (c), integrated intensity ratios (b') and (c') and linewidths (b"') and (c") for selected bands K1 and K2. Solid lines are guides for the eye only. Vertical dotted lines indicate temperature range in which the discontinuity occurs (marked by bold dotted line). The temperature dependency of spectral characteristic for bands G and H are given in Supplemental material.

As well as similarities in both bands patterns and temperature behaviour, the Raman spectra of all three materials evolve continuously without drastic spectral changes, which would indicate a structural phase transition. Nevertheless, it has been shown in the past, that more detailed analysis of the spectra in terms of monitoring position, intensity and linewidth of different bands is useful for identifying more subtle structural rearrangements [55]. Taking this into consideration, the temperature-evolution of the spectral characteristic for selected bands of all three materials is presented in figures $8(b)-(c '), 9(b)-(c ')$ and 10(b)-(c'). The choice has been guided by identifying isolated bands and considering in more detail high-frequency bands which are often particularly sensitive to structural changes [28]. An illustration of this is the selection of the single-line band at $331 \mathrm{~cm}^{-1}$ for $\mathrm{BaMgF}_{4}$ (labelled C in figure 8(a)) which remains well isolated across the whole temperature range. While this band has been analysed for all three samples, different additional bands have been selected depending on the actual spectral signature of the material. 
Detailed spectral analysis illustrated in figures 8(b)-(c"), 9(b)-(c") and 10(b)-(c") provides corroborating evidence for subtle but significant spectral anomalies in the range from 200 to $250 \mathrm{~K}$. Observed anomalies are more or less pronounced depending on the material and the band investigated. The identification of a critical temperature is dependent on the band and material under consideration. Taking the example of $\mathrm{BaMgF}_{4}$, we observe the most significant changes in the temperature evolution of the band position, as clear from the analysis of selected bands C and D1 (figure 8). For the doped samples, $\mathrm{BaMg}_{0.999} \mathrm{Mn}_{0.001} \mathrm{~F}_{4}$ and $\mathrm{BaMg}_{0.995} \mathrm{Ni}_{0.005} \mathrm{~F}_{4}$ the spectral changes are more pronounced and are observed not only in the band position, but also in the intensity ratio and linewidth as illustrated in figures 9 and 10 , respectively.

(a)

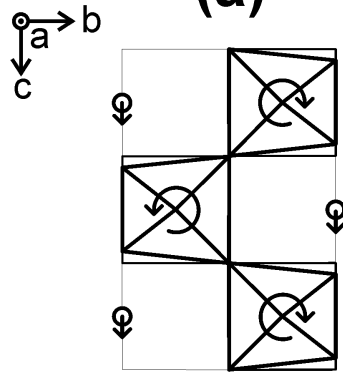

paraelectric phase (hypothetical)
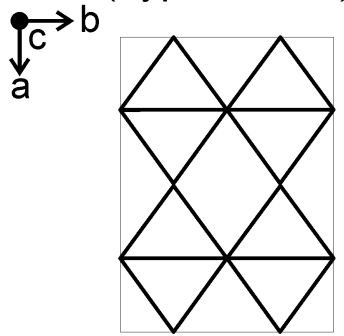

(b)

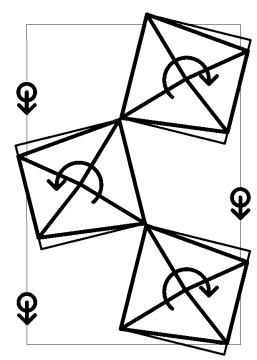

ferroelectric phase (not observed)

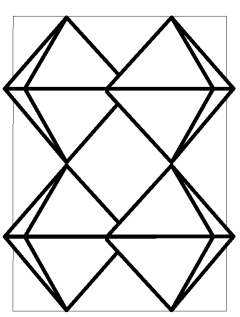

(c)

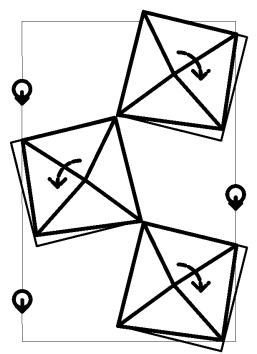

ferroelectric phase

(observed)
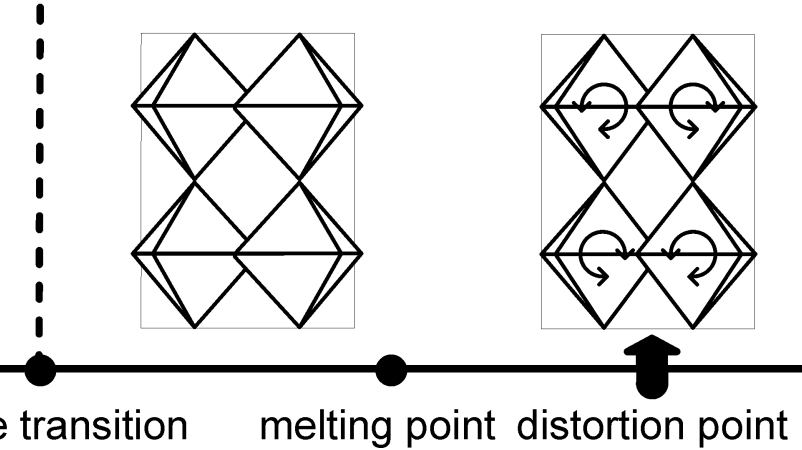

\section{)}

phase transition melting point distortion point

(d)

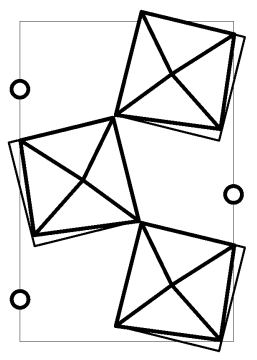




\section{Molecular dynamics in geometric ferroelectric fluorides}

recently have been reached [35-40]. Despite the subtle character of the observations, their consistency across multiple experimental techniques and various samples of $\mathrm{BaMF}_{4}$ materials provides strong evidence in favour of the proposed changes in the structure.

The structure of $\mathrm{BaMF}_{4}$ in the hypothetical paraelectric phase consists of corner-sharing fluoride octahedra, which form sheets perpendicular to $b$ direction (figure 11(a)) [18, 20, 41]. The MF-M chains are linear both along $c$ and $b$ directions (figure 11(a)) [18, 20, 41]. In the paraelectric phase, octahedra do not have a highly symmetric regular shape but are trapezoidal as assumed in a realistic model of close packing (figure 11(a)) [20]. The transition from paraelectric to ferroelectric phase involves rotation and distortion of octahedra from their initial configuration and is accompanied by shifts of $\mathrm{Ba}^{2+}$ and $\mathrm{M}^{2+}$ ions as shown schematically in figure 11(b) [20]. Such behaviour, continues as the temperature is lowered resulting in a more compact arrangements of ions and a gain of energy by the crystalline lattice (figure 11(b)) [20].

The paraelectric phase in $\mathrm{BaMF}_{4}$ materials has never been observed directly since the Curie point is above the melting temperature [16]. However, the paraelectric - ferroelectric transition and its mechanism have been strongly evidenced by both experimental and theoretical studies $[16,20,21$, 24]. There is also strong experimental evidence of the existence of the "soft" Raman bands which disappear at the Curie point [24, 34]. Previously published EPR data also predicts the phase transition from the rotation patter of the main axis of the ZFS tensor [33].

During further cooling short-range interactions between ions (close packing) will slow down and finally prevent continuous rotation and continuous geometrical distortion of octahedra by "locking" their movement in the $c b$ plane (figure 11(d)). The "locking" point will strongly depend on the size of the ion occupying the centre of octahedra because it determines the initial level of distortion at a given temperature. Now, to reduce further the crystal energy, octahedra have to tilt around a new axis perpendicular to the $a$ direction (figure 11(d)). As such a change of rotation is not coherent over the whole sample, it initially induces disorder (dynamic clusters of librating octahedra) rather than a phase transition [25-28, 34]. For high level of initial distortion, as observed only in $\mathrm{BaMnF}_{4}$, a further decrease of temperature leads to the coherent rotation and the incommensurate commensurate phase transition at $250 \mathrm{~K}$ [24].

Our data reflect the above consideration. The temperature dependence of the ZFS parameter $|D|$ (figure 5(a) and (b)) is strongly dependent on molecular dynamics of the lattice because both thermal expansion and phonon contributions are present and approximately equally important [29, 53]. The assumption is justified in the case of geometric ferroelectric fluorides because both thermal expansion and dynamics of phonons are strongly related to the unique structure and its dynamic manifested in rotation and continuous distortion of octahedra. 
In such a case, an increase of $|D|$ when reducing the temperature from $700 \mathrm{~K}$ to room temperature (figure 5(a) and (b)) can be related to the rotation of the octahedra around an $a$ axis and associated with it continuous small changes in the geometry (figure 11). This is supported by the corresponding decrease of $|E|$ (figure 5(a') and (b')), which implies that a more axial symmetry is prevalent at lower temperatures [56]. This continuous behaviour of $|D|$ and $|E|$ likely corresponds to the observed temperature dependency of the phonon frequency (figures 7, 8, 9, 10).

At $\sim 300 \mathrm{~K}$ for $\mathrm{BaZnF}_{4}$ and $240 \mathrm{~K}$ for $\mathrm{BaMgF}_{4}$ a change in the temperature dependencies of $|D|$ and $|E|$ are observed (figure 5). The most likely mechanism of those changes is the discontinuous distortion of the geometry of the octahedra which originates in its rotation around a new direction (figure 11(c)). Additionally, below $\sim 300$ and $\sim 240 \mathrm{~K}$ (for $\mathrm{BaZnF}_{4}$ and $\mathrm{BaMgF}_{4}$ respectively), the shape of the temperature dependencies of $|D|$ and $|E|$ will change. This change is due to the temperature dependence of the ZFS mechanism being more strongly dependent on the couplings with phonons rather than thermal expansion, which at lower temperatures is expected to be less efficient than at high temperatures $[29,53]$.

The discontinuous distortion mechanism is further supported by our ${ }^{19} \mathrm{~F}$ MAS NMR data. The temperature dependency of the relative differences in the chemical shift $\Delta \delta_{\text {iso }}{ }^{i j}$ for $\mathrm{BaMgF}_{4}$ (figure 3(b)) shows a discontinuity at approximately the same temperature as the EPR data. This discontinuity is caused by a discontinuous change in the fluorine coordination sphere and can be directly related to a discontinuous change of the geometry of the fluorine octahedra. Since the main change involves $\mathrm{F}(1), \mathrm{F}(2)$ and $\mathrm{F}(3)$ fluorine nuclei (changes in bond lengths and their angles) (figure 1) and it is not related to continuous rotation it is most likely that the origin of this discontinuous distortion is rotation of the octahedra about a new axis (figure 11(c)). The temperature of the observed change in the NMR data $220 \pm 20 \mathrm{~K}$ corresponds, within experimental error to the temperature at which changes in the ZFS parameters are observed $(240 \pm 20 \mathrm{~K})$.

Our Raman data are in agreement with this hypothesis and provide further evidence for the discontinuous distortion mechanism. Subtle anomalies in the frequency and linewidth of some of the high energy bands in $\mathrm{BaMgF}_{4}$ (figure 8), $\mathrm{BaMg}_{0.999} \mathrm{Mn}_{0.001} \mathrm{~F}_{4}$ (figure 9), and $\mathrm{BaMg}_{0.995} \mathrm{Ni}_{0.005} \mathrm{~F}_{4}$ (figure 10) are observed between $200 \mathrm{~K}$ and $250 \mathrm{~K}$ and reflect their sensitivity to small modifications of the geometry of the octahedra (see previous section). Such small changes in the geometry of the octahedra are not expected to have any significant effect on the softening or hardening of bands related to their collective rotation around the $a$ axis. Instead, they will have a much more significant influence on phonons related to vibration of the atoms forming the octahedra and will be manifested in anomalies of the frequencies and possibly linewidths. 
At the same time, observed anomalies cannot be explained by the presence of dopants or defects, because they have been observed for undoped $\left(\mathrm{BaMgF}_{4}\right.$, figure 8$)$ and doped samples $\left(\mathrm{BaMg}_{0.999} \mathrm{Mn}_{0.001} \mathrm{~F}_{4}\right.$, figure 9 and $\mathrm{BaMg}_{0.999} \mathrm{Ni}_{0.001} \mathrm{~F}_{4}$, figure 10), with different level of defects.

The observed difference in the temperatures at which the distortion occurs, $(300 \mathrm{~K}$ for $\mathrm{BaZnF}_{4}$ and $240 \mathrm{~K}$ for $\left.\mathrm{BaMgF}_{4}\right)$ also supports the relation with the geometry of the octahedra (compare figures 5, 7 and 8). This difference in the temperature can be related to the different ionic radii of $\mathrm{Mg}^{2+}$ and $\mathrm{Zn}^{2+}(0.66 \AA$ and $0.74 \AA$, respectively) and as a result have different degrees of initial lattice distortion at the same temperature below the Curie point. For the more distorted structure (i.e. $\mathrm{BaZnF}_{4}$ ) changes in the dynamics have to occur earlier, which is in agreement with the $\sim 60 \mathrm{~K}$ observed difference between $\mathrm{BaZnF}_{4}$ and $\mathrm{BaMgF}_{4}$.

We believe that the observed change in the geometry opens the way for the octahedra to rotate around a different, more favourable direction and as a result it could be a precursor of some form of ordering. Rotation about the $a$ axis still takes place, but with significantly less effect on the geometry of the octahedra and finally stops, reaching a "locking" point. Our suggested hypothesis is in agreement with an idea of the formation of the dynamic clusters as the precursors of the instability which lead to doubling of the unit cell dimension along the $a$ axis and a commensurate incommensurate phase transition [25-28]. Such a phase transition is not observed in $\mathrm{BaMgF}_{4}$ and $\mathrm{BaZnF}_{4}$ because the level of lattice distortion is insufficient to force long-range ordering in the $a c$ planes even at cryogenic temperatures. Only for $\mathrm{BaMnF}_{4}$, where structural distortion is highest, in the family, can this mechanism lead to the well documented phase transition [24].

\section{Conclusions}

In these studies, we applied successfully magnetic resonance techniques (NMR and EPR) and Raman scattering to elucidate in detail local nuclear and electronic structures as well as low temperature changes in molecular dynamics of selected geometric ferroelectric fluorides.

The ${ }^{25} \mathrm{Mg}$ isotropic chemical shift $\delta_{\text {iso, }}$ quadrupolar coupling constants $\left(\mathrm{C}_{\mathrm{q}}\right)$ and asymmetry parameters $(\eta)$ reflect the distorted geometry of the $\mathrm{Mg}^{2+}$ octahedral coordination. For $\mathrm{Mn}^{2+}$, which replace $\mathrm{Mg}^{2+}$ during doping, Zero Field Splitting parameters $|D|$ and $|E|$ are consistent with distorted axial symmetry, at low temperatures and nearly rhombic, at high temperatures of octahedral $\mathrm{Mn}^{2+}$ coordination. The observed high resolution of NMR, EPR and phonon spectra are consistent with the highly ordered crystallographic structure.

The most important result is the discovery of the discontinuous change in the structural distortion of the fluoride octahedra, which occurs at $\sim 300 \mathrm{~K}$ for $\mathrm{BaZnF}_{4}$ and $\sim 240 \mathrm{~K}$ for $\mathrm{BaMgF}_{4}$. This distortion opens the way for the octahedra to rotate around a new axis approximately 
perpendicular to that related to the paraelectric - ferroelectric phase transition. The temperature at which this change occurs depends on the ionic radius of the central $\mathrm{M}^{2+}$ ion of the fluorine octahedra.

The detection and analysis of these very subtle changes was only possible thanks to application of different complementary experimental techniques. Combined analysis of the temperature changes in the Zero Field Splitting parameters $|D|$ and $|E|$, observed discontinuities in the temperature dependence of isotropic chemical shift and anomalies in the frequencies and linewidths of some high frequency Raman bands suggest that this geometrical change plays an important role in modifying the lattice dynamics as an initial step in the creation of dynamic order in the structure.

\section{Acknowledgments}

We thank Prof David Collison for valuable discussions and comments during the course of this work.

We also thank the University of Warwick Research Development Fund (project No. RD08145) for providing partial financial support and British Council for providing travel and subsistence funds in the frame of Alliance 2010 Franco - British Research Partnership programme.

The X-ray diffractormeter and the $500 \mathrm{MHz}$ NMR spectrometer used in this research were obtained, through Birmingham Science City Advanced Materials Projects 1 and 2 and Hydrogen Energy Project, with support from Advantage West Midlands (AWM) and European Regional Development Fund (ERDF). The Varian $600 \mathrm{MHz}$ NMR spectrometer was purchased for an EPSRC (UK) Basic Technology Project, EP/D045967.

\section{References:}

[1] Scott J F 2007 Science 315 954-9

[2] Ziebowicz B, Szewieczek D, Dobrzanski L A 2007 Journal of Achievements in Materials and Manufacture Engineering 20 207-10

[3] Egami T 2007 Annu. Rev. Mater. Res. 37 297-315

[4] Eerenstain W, Mathur N D, Scott J F 2006 Nature Mat. 442 759-65

[5] Hill N A 2000 J. Phys. Chem. B 104 6694-709

[6] Fiebig M 2005 J. Phys. D: Appl. Phys. 38 R123-52

[7] Van Aken B B, Palstra T T M, Filippetti M A, Spaldin N A 2004 Nature Mat. 3 164-70

[8] Ivanov V Y, Mukhin A A, Travkin V D, Prokhorov A S, Popov Y F, Kadomtseva A M, Voroblev G P, Kamilov K I, Balbashov A M 2006 Phys. Stat. Sol. 243 107-11

[9] Lee S, Pirogov A, Kang M, Jang K-H, Yonemura M, Kamiyama T, Cheong S-W, Gozzo F, Shin N, Kimura H, Noda Y, Park J-G 2008 Nature 451805

[10] Fiebig M, Lottermoser Th, Kneip M K, Bayer M 2006 J. Appl. Phys. 99 08E3021-5 
[11] Sato T J, Lee S-H, Katsufuji T, Masaki M, Park S, Copley J R D, Takagi H 2003 Phys. Rev. $680144321-5$

[12] Scott J F, Blinc R 2011 J. Phys.: Condens. Matter 23 1132021-17

[13] Scott J F 1977 Phys. Rev. B 16 2329-31

Fox D L, Tilley D R, Scott J F, Guggenheim H J 1980 Phys. Rev. B 21 2926-36

Tilley D R, Scott J F 1982 Phys. Rev. B 25 3251-60

[14] Fox D L, Scott J F 1977 J. Phys. C: Solid Stat. Phys. 10 L329-31

[15] Ederer C, Spaldin N A 2006 Phys. Rev. B 74 020401R1-4

[16] Eibschutz M, Guggenheim H J, Wemple S H, Camlibel I, DiDomenico M Jr 1969 Phys. Lett. 29A 409-410

DiDomenico M Jr, Eibschiitz M, Guggenheim H J, Camlibel I 1969 Solid Stat. Commun. 7 $1119-22$

Ederer C, Spaldin N A 2006 Phys. Rev. B 74 0241021-8

[17] Bergman J G, Crane G R, Guggenheim H 1975 J. Appl. Phys. 46 4645-6

Asahi T, Tomizawa M, Kobayashi J, Kleemann W 1992 Phys. Rev. B 45 1971-87

Víllora E G, Shimamura K, Jing F, Medvedev A, Takekawa S, Kitamura K 2007 Appl. Phys. Lett. 90 1929091-3

Tong Y, Meng X Y, Wang Z Z, Chena C, Lee M - H 2005 J. Appl. Phys. 98 0335041-7

[18] Gingl F 1997 Z. Anorg. Allg. Chem. 623 705-9

[19] Eibschtitz M, Guggenheim H J 1968 Solid Stat. Commun. 6 737-9

[20] Keve E T, Abrahams S C, Bernstein J L 1969 J. Chem. Phys. 51 4928-36

Keve E T, Abrahams S C, Bernstein J L 1970 J. Chem. Phys. 53 3279-87

[21] Flocken J, Mo Z, Mei W N, Hardy J R, Hatch D M 1994 Phys. Rev. B 49 5812-16

[22] Cox D E, Shapiro S M, Cowley R A, Eibschutz M, Guggenheim H J 1979 Phys. Rev. B 19 5754-72

Yoshimuraa M, Hidakab M, Mizushimac T, Sakuraic J, Tsuboid T, Kleemann W $2006 \mathrm{~J}$. Mag. Mag. Mater. 299 404-411

Veira J R, Argyriou D N, Kiefer K, Wolter A U B, Alber D, Meissner M, Almairac R, Reehuis M, Bordallo H M 2008 Phys. Rev. B 78 0541041-6

[23] Cox D E, Eibschutz M, Guggenheim H J, Holmes L 1970 J. Appl. Phys. 41 943-5

[24] Scott J F 1979 Rep. Prog. Phys. 12 1056-84

Ryan J F, Scott J F 1974 Solid Stat. Commun. 14 5-9

Bechtle D W, Scott J F, Lockwood D J 1978 Phys. Rev. B 18 6213-29

Lavrencic B B, Scott J F 1981 Phys. Rev. B 24 2711-7

Lockwood D J, Murray A F, Rowell N L 1981 J. Phys. C: Solid State Phys. 14 753-72 
Ryan T W 1986 J. Phys. C: Solid State Phys. 19 1097-106

Sciau P, Lapasset J, Grebille D, Berar J F 1988 Acta Cryst. B 44 108-16

Yoshimura M, Hidaka M 2005 J. Phys. Soc. Jap. 74 1181-9

[25] Bordallo H N, Bulou A, Almairac R, Nouet J 1994 J. Phys.: Condens. Matter 6 10365-76

[26] Almairac R, Bordallo H N, Bulou A, Nouet J 1995 Phys. Rev. B 52 9370-6

[27] Almairac R, Bordallo H N, Bulou A, Nouet J, Currat R 1997 Phys. Rev. B 55 8249-56

[28] Bordallo H N, Almairac R, Bulou A, Nouet J 1996 J. Phys.: Condens. Matter 8 4993-5005

[29] Wan K L, Hutton S L, Drumheller J E 1987 J. Chem. Phys. 86 3801-3

[30] Cai S-H, Yu X-Y, Chen Z, Wan H-L 2003 Mag. Res. Chem. 41 902-7

Body M, Silly G, Legein C, Buzar J-Y 2005 J. Phys. Chem. B 109 10270-8

Zheng A, Liu S-B, Deng F 2009 J. Phys. Chem. C 113 15018-23

[31] Recher K, Wallrafen F, Buscher R, Lehmann G 1981 Phys. Status Solidi b 107 699-706

Yi-Yang Z 1988 J. Phys. C: Solid State Phys. 21 5547-53

Dance J M, Boireau A, Le Lirzin A, Lestienne B 1994 Solid State Commun. 91 475-9

[32] Rey J M, Bill H, Lovy D, Hagemann H 1998 Journal of Alloys and Compounds 268 60-5

Yamaga M, Hattori K, Kodama N, Ishizawa N, Honda M, Shimamura K, Fukuda T $2001 \mathrm{~J}$.

Phys.: Condens. Matter 13 10811-24

[33] Fukui M, Chikushi S, Abe R 1980 J. Phys. Soc. Jap. 48 1196-201

[34] Quilichini M, Ryan J F, Scott J F 1975 Solid State Commun. 16 471-5

[35] Wind M, Saalwaechter K, Wiesler U-M, Muellen K, H. Spiess H W 2002 Macromol. 35 10071-86

[36] Krzyminiewski R, Bielewicz-Mordalska A, Kowalczyk R M 1998 J. Mag. Res. 135 76-81

[37] Bismayer U 1990 Phase Transitions 27 211-267

[38] Bakhmutov V I 2010 Chem. Rev. ASAP Article DOI: 10.1021/cr100144r

[39] Cameron T S, Decken A, Kowalczyk R M, McInnes E J L, Passmore J, Rawson J M, Shuvaev K V, Thompson L K 2006 Chem. Commun. 2277-79

[40] Iliev M N, Litvinchuk A P, Abrashev M V, Popov V N, Cmaidalka J, Lorenz B, Meng R L 2004 Phys. Rev. B 69172301

Girardot C, Kreisel J, Pignard S, Caillault N, F. Weiss F 2008 Phys. Rev. B 78104101

[41] Posse J M, Grzechnik A, Friese K 2009 Acta Cryst. B 65 576-86

[42] Kunwar A C, Turner G L, Oldfield E 1986 J. Magn. Reson. 69 124-7

[43] Bodart P R, Amoureux, Dumazy J P, Lefort R, 2000 Mol. Phys. 98 1545-51

[44] Bak M, Rasmussen J T, Nielsen N C 2000 J. Mag. Res. 147 296-330

[45] Clark S J, Segall M D, Pickard C J, Hasnip P J, Probert M J, Refson K, Payne M C 2005 Zeitschrift für Kristallographie 220 567-70 
[46] Stoll S, Schweiger A 2006 J. Magn. Reson. 178 42-55

[47] Gross U, Ruediger S, Grimmer A-R, Kemnitz E 2002 Journal Fluorine Chemistry 115 193-9

[48] Bureau B, Silly G, Emery J, Buzaré J-Y 1999 Chem. Phys. 249 89-104

Body M, Silly G, Legein C, Buzaré J-Y 2005 J. Phys. Chem. B 109 10270-8

[49] Bastow T J 2002 Solid State Commun. 124 269-73

Freitas J C C, Wong A, Smith M E 2009 Magn. Reson. Chem. 47 9-15

[50] Bleich H E, Redfield A G 1977 J. Chem. Phys. 67 5040-7

Dupree R, Smith M E 1988 J. Chem. Soc., Chem. Commun. 1483-5

Bastow T J 1991 Solid State Commun. 77 547-8

[51] Cahill L S, Hanna J V, Wong A, Freitas J C C, Yates J R, Harris R K, Smith M E 2009 Chem. Eur. J. 15 9785-98

Widdifield C M, Bryce D L 2009 Phys. Chem. Chem. Phys. 11 7120-2

[52] Yosida T, Aoki H, Takeuchi H, Arakawa M, Horai K 1991 J. Phys. Soc. Jap. 60 625-35

[53] Shrivastava K N 1969 Phys. Rev. 187 446-50

Wen-Chen Z, Shao-Yi W 1996 Phys. Rev. B 54 1117-22

[54] Fukui M, Hirose T 1980 J. Phys. Soc. Jap. 49 1399-404

[55] U. Bismayer U 1990 Phase Transitions 27 211-67

Salje E K H, Bismayer U 1997 Phase Transitions 63 1-75

Kreisel J, Glazer A M, Jones G, Thomas P A, Abello L, Lucazeau G 2000 J. Phys.: Condens. Matter 12 3267-80

[56] Mabbs F, Collison D 1992 EPR of transition metal compounds (Elsevier) 